\title{
The Crystal Structure of UehA in Complex with Ectoine-A Comparison with Other TRAP-T Binding Proteins
}

Justin Lecher $^{1} \dagger$, Marco Pittelkow ${ }^{2} \dagger$, Silke Zobel ${ }^{1}$, Jan Bursy ${ }^{2}$,
${ }_{5}$ Tobias Bönig $^{2}$, Sander H.J. Smits ${ }^{1}$, Lutz Schmitt ${ }^{1 *}$ and Erhard Bremer ${ }^{2}$

${ }^{1}$ Institute of Biochemistry,

23 Heinrich-Heine-University

84 Duesseldorf, Universitätsstrasse

95 1, 40225 Duesseldorf, Germany

${ }_{11}^{26}$ Laboratory for Microbiology,

${ }_{12}^{27}$ Department of Biology,

${ }_{13}^{29}$ Philipps University Marburg,

${ }_{14}^{29}$ Karl-von-Frisch Strasse 8,

15 D-35032 Marburg, Germany

32 Received 20 February 2009;

䪦 received in revised form

\$9 30 March 2009;

影 accepted 31 March 2009

Edited by R. Huber

\begin{abstract}
Substrate-binding proteins or extracellular solute receptors (ESRs) are components of both ABC (ATP binding cassette) and TRAP-T (tripartite ATP-independent periplasmic transporter). The TRAP-T system UehABC from Silicibacter pomeroyi DSS-3 imports the compatible solutes ectoine and 5-hydroxyectoine as nutrients. UehA, the ESR of the UehABC operon, binds both ectoine and 5-hydroxyectoine with high affinity $\left(K_{\mathrm{d}}\right.$ values of $1.4 \pm 0.1$ and $1.1 \pm 0.1 \mu \mathrm{M}$, respectively) and delivers them to the TRAP-T complex. The crystal structure of UehA in complex with ectoine was determined at 2.9-A resolution and revealed an overall fold common for all ESR proteins from TRAP systems determined so far. A comparison of the recently described structure of TeaA from Halomonas elongata and an ectoine-binding protein (EhuB) from an $\mathrm{ABC}$ transporter revealed a conserved ligand binding mode that involves both directed and cation-pi interactions. Furthermore, a comparison with other known TRAP-T ESRs revealed a helix that might act as a selectivity filter imposing restraints on the ESRs that fine-tune ligand recognition and binding and finally might determine the selection of the cognate substrate.
\end{abstract}

(C) 2009 Published by Elsevier Ltd.

Keywords: TRAP transporters; periplasmic binding proteins; ectoine; compatible solutes affinity and specificity by the SBP that is either 56 located in the periplasm of Gram-negative bacteria 57 or anchored at the cell surface of Gram-positive 58 bacteria or archaea. ${ }^{1}$ After initial ligand binding, the 59 substrate-loaded SBP delivers the ligand to the 60 designated membrane-embedded components of 61 the ABC transporter for subsequent ATP-dependent 62 import. The SBP is essential for substrate uptake, 63 imposes directionality on a given $\mathrm{ABC}$ transporter 64 and regulates the ATPase activity of the nucleotide- 65 binding protein. ${ }^{3}$

In a pioneering study, Forward et al ${ }^{4}$ identified a 67 novel multicomponent transport system in the 68 photosynthetic bacterium Rhodobacter capsulatus 69 that contained an ESR, but this transport system, 70 in striking contrast to $\mathrm{ABC}$ transporters, operated 71 independently of ATP hydrolysis. These transpor- 72 ters were subsequently named TRAP-Ts (tripartite 73 ATP-independent periplasmic transporters), ${ }^{5}$ and 74 they use electrochemical ion gradients $\left(\mathrm{H}^{+}\right.$or $\left.\mathrm{Na}^{+}\right) 75$ to fuel the uphill transport of substrates. TRAP 76 systems are thus secondary active transporters but 77 require an ESR for proper functioning. ${ }^{6-9}$ In the 78 
following, we use the term "SBP" for the binding proteins of $\mathrm{ABC}$ transport systems and the term "ESR" for the binding protein of TRAP-Ts to differentiate between both families.

Systematic genome-wide bioinformatic analysis revealed a widespread distribution of TRAP-Ts within the domains of bacteria and archaea, with an increased number of TRAP-type transporters found in microorganisms living in saline environments. ${ }^{8}$ Use of electrochemical sodium gradients to energize TRAP-Ts would be advantageous to marine microorganisms because it would allow these bacteria to import substrates at a lower energetic cost than that required for the functioning of an $\mathrm{ABC}$ transporter uptake system that depends on ATP hydrolysis. ${ }^{8}$ In vitro reconstitution of the sialic acid-specific SiaPQM system from the human pathogen Haemophilus influenzae demonstrated that a sodium gradient is used to fuel substrate import by a TRAP-type system. ${ }^{9}$

TRAP-Ts, as exemplified by the C4-dicarboxylate import system (DctPQM) from $R$. capsulatus, ${ }^{4,5}$ usually comprise three components: a large membrane-embedded subunit (DctM), a small membraneembedded subunit (DctQ) and an extracellular SBP (DctP), the ESR. The large subunit probably forms the solute translocation channel ${ }^{7,8}$ and usually comprises 12 transmembrane-spanning segments. The small subunit contains 4 transmembrane-spanning segments. Both proteins are required for the functioning of the TRAP-T, but the precise role of the single subunits in the overall transport process is still unclear $^{7,8}$ In some TRAP systems, such as SiaPQM, the large and small membrane subunits are fused in a single polypeptide. ${ }^{9}$ The best studied components of TRAP systems are the soluble ESRs. ${ }^{10-16}$ In vitro studies with the SiaPQM system have recently shown that the SBP imposes directionality on the overall transport process, ${ }^{9}$ but it is so far unclear whether the observed binding protein-dependent export reaction of a TRAP system is of physiological relevance.

Several ESR crystal structures, with or without bound ligand, have advanced our understanding of the ESR proteins of TRAP systems. These crystal structures include SiaP from $H$. influenzae, ${ }^{12,17}$ specific for sialic acids; TakP from Rhodobacter sphaeroides, ${ }^{13}$ specific for $\alpha$-keto acids; DctP6 and DctP7 from Bordetella pertussis, ${ }^{14}$ both specific for pyroglutamic acid; TeaA from Halomonas elongata, ${ }^{15}$ specific for ectoine and 5-hydroxyectoine; and the structure of an ESR from the hyperthermophile Thermotoga maritima, ${ }^{16}$ with a serendipitously captured and chemically poor defined substrate. Structural analysis of these ESRs revealed a common protein fold and the same bilobal organization observed for SBPs. ${ }^{18,19}$ Furthermore, the structural analysis of the SiaP and TakP proteins in the presence and in the absence of their specific ligands suggested that both proteins follow the "Venus flytrap" mechanism of SBPs for capturing of the cognate ligand. ${ }^{12,13,19}$ This mechanism proposes a constant opening-and-closing motion of the bilobal
SBPs of ABC transporters. The binding of the ligand 142 shifts the equilibrium between these various con- 143 formations toward the closed state in which the 144 substrate has been trapped by the SBP. ${ }^{18}$ A flexible 145 linker connects the two domains of the SBP and 146 permits a hinge-bending movement that leads to 147 rigid-body motions conducted by both domains of 148 the SBP. As a consequence, the ligand-binding site is 149 dynamically formed with contributions of both 150 domains of the SBP and, concomitantly, the ligand 151 is trapped within the deep cleft formed by the two 152 domains of the SBP. The structural investigations 153 support the idea that the TRAP-associated ESRs and 154 ABC-associated SBPs are functionally similar with 155 respect to their role in transport. $8,12,17 \quad 156$

In this study, we focused on the ectoine/5- 157 hydroxyectoine ligand-binding protein UehA from 158 Silicibacter pomeroyi DSS $-3,{ }^{20}$ a member of the 159 Roseobacter lineage of marine $\alpha$-proteobacteria that 160 plays an important role for global carbon and sulfur 161 cycles in oceans. ${ }^{21}$ S. pomeroyi DSS-3 possesses an 162 uncommonly high number of TRAP transport 163 systems, ${ }^{8}$ one of which is the UehABC importer. 164 Herein, we show that ectoine and 5-hydroxyectoine 165 are two natural ligands of UehA. Both ligands are 166 compatible solutes that are widely used by micro- 167 organisms to offset the detrimental effects of high 168 salinity on cell physiology. ${ }^{22}$ However, S. pomeroyi 169 DSS-3 does not use these tetrahydropyrimidines as 170 osmoprotectants and instead employs them as 171 nutrients.

We determined the crystal structure of the UehA- 173 ectoine complex and compared the overall fold of 174 UehA and the architecture of the ligand-binding site 175 with those of the TeaA-ectoine complex. ${ }^{15}$ TeaA is 176 an ESR from an ectoine/5-hydroxyectoine-specific 177 TRAP-T involved in osmoprotection in the moderate 178 halophile H. elongata. ${ }^{23}$ Furthermore, we compared 179 the UehA-ectoine complex with the crystal structure 180 of an ectoine and 5-hydroxyectoine-specific SBP 181 (EhuB). ${ }^{24}$ Finally, a comparison of all available 182 crystal structures of ESRs from TRAP systems 183 revealed a helix within these ligand-binding pro- 184 teins that probably plays an important role in fine- 185 tuning and determining the substrate selectivity of 186 TRAP-associated ESRs.

\section{A putative TRAP-T for ectoine and 5-hydroxyectoine in S. pomeroyi DSS-3}

In marine waters, the nutrient concentrations are 191 generally low and microorganisms must therefore 192 possess effective mechanisms to scavenge organic 193 compounds from such an oligotrophic environment. 194 The marine bacterium S. pomeroyi DSS-3 is a member 195 of the ecologically important Roseobacter lineage of 196 $\alpha$-proteobacteria, ${ }^{21}$ and its genome sequence has 197 revealed a number of adaptations to nutrient-poor 198 environments. Among these is the occurrence of an 199 
uncommonly large number of TRAP-Ts. ${ }^{8,20}$ We found that S. pomeroyi DSS-3 can efficiently use the tetrahydropyrimidines ectoine and 5-hydroxyectoine (see Fig. 3 for their chemical structures) as sole nitrogen and carbon sources. Hence, an uptake system for ectoine and 5-hydroxyectoine must operate in S. pomeroyi DSS-3 to provide the cell with these compounds as nutrients. Surprisingly, both compatible solutes did not function as osmostress protectants in S. pomeroyi DSS-3 (J.B., T.B. and E.B., unpublished results) as they commonly do in many bacterial species. ${ }^{22,25-28}$

Inspection of the $S$. pomeroyi DSS-3 genome sequence $^{20}$ identified a large gene cluster (Fig. 1a) whose predicted proteins encode a TRAP-type import system for ectoine and 5-hydroxyectoine and enzymes mediating the degradation of these compounds for nutritional purposes. The components of the TRAP-type transporter UehABC (Ueh: $u$ ptake of ectoine and hydroxyectoine) are related to the subunits of a functionally characterized ectoine/ 5-hydroxyectoine TRAP transport system (TeaABC) from the moderate halophile $H$. elongata. ${ }^{23}$ The TeaABC system serves for the osmotically regulated import of ectoine and 5-hydroxyectoine as osmoprotectants and as a salvaging system for ectoine/ 5-hydroxyectoine that leaks from the ectoine/5hydroxyectoine-producing $\mathrm{H}$. elongata cells. ${ }^{23}$ Genes encoded downstream of $u e h A B C$ are referred to as eut $A B C D E$ (Eut: ectoine utilization) and spo1137/ 229 spo1136 (Fig. 1a). The EutABCDE, Spo1137 and 230 Spo1136 proteins are homologous to ectoine-induced 231 proteins found in Sinorhizobium meliloti when this soil 232 bacterium is grown in the presence of ectoine, but 233 their physiological role in ectoine/5-hydroxyectoine 234 degradation by S. meliloti has been studied only to 235 some extent. ${ }^{29}$

The UehA protein of S. pomeroyi DSS-3 is 237 predicted to encode a periplasmic ESR with 58\% 238 amino acid sequence identity to the functionally 239 characterized ectoine/5-hydroxyectoine-binding 240 protein TeaA from $H$. elongata. ${ }^{15}$ The small integral 241 membrane component (UehB) of the TRAP system 242 exhibits $56 \%$ amino acid sequence identity to TeaB, 243 and the large integral membrane component (UehC) 244 exhibits $74 \%$ amino acid sequence identity to TeaC. 245 Prediction of the transmembrane topology ${ }^{30} 246$ revealed 4 and 12 membrane-spanning segments 247 for the UehB and UehC proteins, respectively, a 248 topological arrangement that is typical for the 249 membrane-embedded large and small subunits of 250 TRAP-type transporters. ${ }^{6-8}$

\section{Substrate-induced uptake of $\left[{ }^{14} \mathrm{C}\right]$ ectoine into S. pomeroyi DSS-3}

The genes encoding transport systems for nutri- 254 ents in microorganisms are often induced by their 255

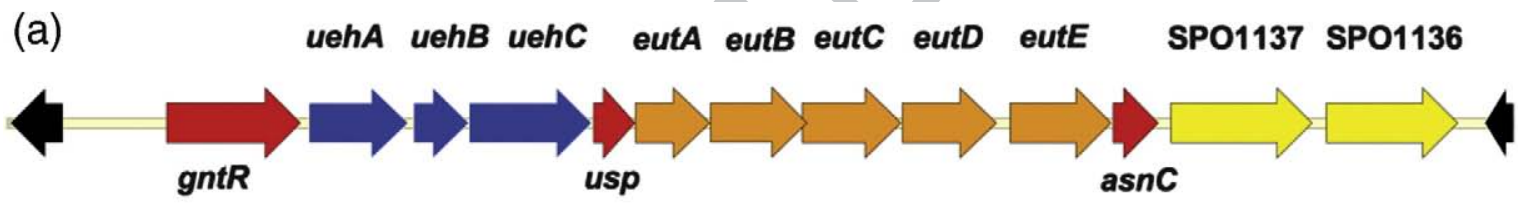

(b)

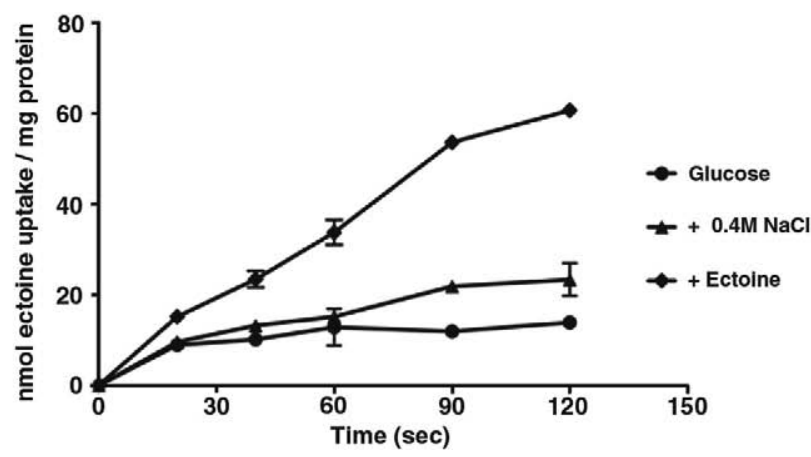

Fig. 1. Genome organization of the ueh-eut region and ectoine uptake of S. pomeroyi DSS-3. (a) Schematic summary of the ueh-eut gene cluster present in S. pomeroyi DSS-3. The uehABC genes encoding the components for a TRAP-type ectoine/5-hydroxyectoine transport system are flanked by genes encoding a GntR-type regulator and a member of the Usp family. The $u s p$ gene is followed by five genes (eutABCDE) that have been implicated in the utilization of ectoine in $S$. meliloti. The eut genes are followed by a gene $(a s n C)$ encoding a regulator for amino acid metabolism in microorganisms. The two genes (spo1137 and spo1136) following asnC possibly encode a succinate-semialdehyde dehydrogenase and an aminotransferase that might also be involved in the degradation of ectoine/5-hydroxyectoine, since such types of proteins are induced when $S$. meliloti is grown in the presence of ectoine. (b) Uptake of $\left[{ }^{14} \mathrm{C}\right]$ ectoine by cells of $S$. pomeroyi DSS-3. Cultures of $S$. pomeroyi DSS-3 were grown in a shaking water bath in basal minimal medium at $30^{\circ} \mathrm{C}$ with either glucose or ectoine as the sole carbon source. A third culture was grown with glucose as the carbon source and an elevated concentration of salt (total concentration of $\mathrm{NaCl}=0.4 \mathrm{M}$ ). $\left[{ }^{14} \mathrm{C}\right]$ Ectoine was added to $2-\mathrm{ml}$ aliquots of the cells at a final substrate concentration of $19 \mu \mathrm{M}$, and scintillation counting was used to monitor the initial uptake of the radiolabeled ectoine. The data shown are the means of two independently grown cultures in which $\left[{ }^{14} \mathrm{C}\right]$ ectoine uptake was measured twice per culture. 
cognate substrates (e.g., the maltose ABC transporter of Escherichia coli). ${ }^{1,2}$ The abovementioned physiological data on the use of ectoine and 5hydroxyectoine as nutrients, but not as osmoprotectants, suggest that ectoine uptake by S. pomeroyi DSS-3 might be induced in cells that are grown in the presence of ectoine. In contrast, ectoine transport should not be enhanced when S. pomeroyi DSS-3 is grown in high-salinity media. S. pomeroyi DSS-3 was grown (i) in minimal medium with glucose as the sole carbon source, (ii) in minimal medium with a total $\mathrm{NaCl}$ content of $0.4 \mathrm{M}$ and (iii) in minimal medium with ectoine as the sole carbon source to test this hypothesis. The various S. pomeroyi DSS-3 cultures were grown at $30^{\circ} \mathrm{C}$ to mid-exponential phase. The uptake of radiolabeled ectoine was subsequently measured over time by the addition of $\left[{ }^{14} \mathrm{C}\right]$ ectoine (final concentration $=19 \mu \mathrm{M}$ ). As shown in Fig. 1b, ectoine uptake was very low in either glucose-grown or high salinity-grown cells but was strongly enhanced when the cells were pregrown with ectoine as the sole carbon source. Hence, S. pomeroyi DSS-3 possesses a high-affinity ectoine transport activity that is substrate inducible but not osmotically stimulated.

\section{Overproduction and purification of the UehA protein}

The sequence relatedness of the UehA ESR from $S$. pomeroyi DSS-3 to the functionally characterized ectoine/5-hydroxyectoine-binding protein TeaA from $H$. elongata ${ }^{15,23}$ suggested to us that the UehA protein might recognize both ectoine and 5-hydroxyectoine as its ligands. The UehA protein was overexpressed with an N-terminal Strep-tag II affinity peptide in E. coli and purified to apparent homogeneity by affinity chromatography on a Strep- 291 Tactin column (Fig. 2a) to verify this. The oligomeric 292 composition of the purified UehA protein was 293 analyzed by size-exclusion chromatography. The 294 UehA protein (calculated molecular mass $=34 \mathrm{kDa}$ ) 295 eluted from the gel-filtration column as a $36-\mathrm{kDa} 296$ protein species (Fig. 2b), indicating that UehA is a 297 monomer in solution. UehA shares this property 298 with all other functionally characterized ESR 299 proteins from TRAP-Ts except for the TakP protein 300 from $R$. sphaeroides and the ESR binding protein 301 from T. maritima, both being dimeric in solution. ${ }^{13,16} 302$

\section{Substrate specificity and binding affinities of UehA for ectoine and 5-hydroxyectoine}

The binding of ectoine and 5-hydroxyectoine by 305 the purified UehA protein was tested through the 306 use of an intrinsic tryptophan fluorescence-based 307 binding assay. ${ }^{24,31,32}$ Addition of increasing concen- 308 trations of either ectoine or 5-hydroxyectoine to the 309 UehA protein resulted in a decrease in the intensity 310 of the tryptophan fluorescence, demonstrating that 311 UehA binds both tetrahydropyrimidines. In con- 312 trast, the addition of the compatible solutes proline, 313 glycine betaine and trehalose did not elicit any 314 change in the intensity of the tryptophan fluores- 315 cence emitted by UehA (data not shown). Conse- 316 quently, UehA from S. pomeroyi DSS-3 is a ligand- 317 binding protein that is specific for ectoine and 5-318 hydroxyectoine as predicted from the amino acid 319 sequence relatedness to TeaA from $H_{\text {. }}$ longata..$^{23} \quad 320$ Based on the 1:1 Langmuir binding isotherm, $K_{d} 321$ values of $1.4 \pm 0.1$ and $1.1 \pm 0.1 \mu \mathrm{M}$ were calculated 322 for ectoine and 5-hydroxyectoine, respectively (Fig. 323 $3 a$ and $b$ ). Hence, the binding affinities of UehA for 324 both ectoine and 5-hydroxyectoine are comparable 325 (a)

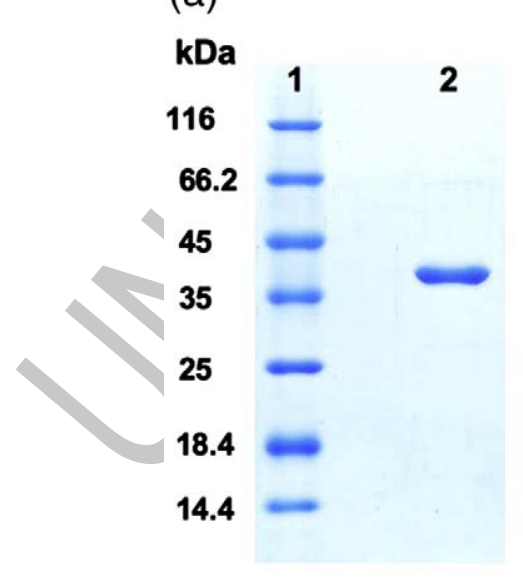

(b)

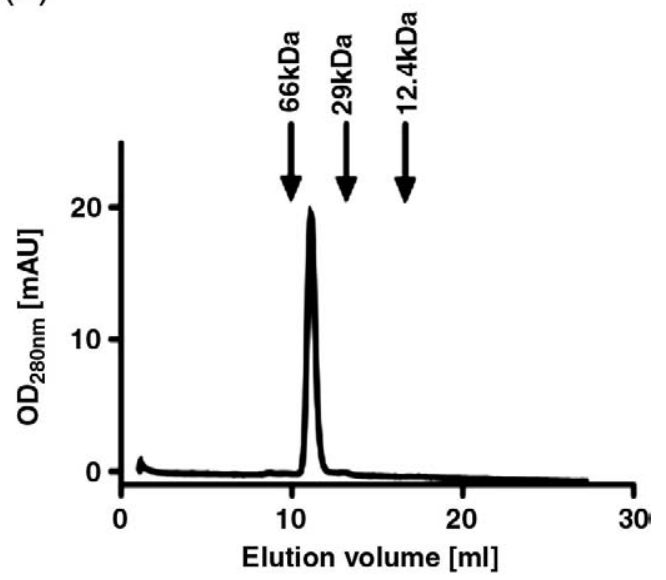

Fig. 2. Analyses of the purified UehA. (a) SDS-PAGE of the purified S. pomeroyi DSS-3 UehA protein produced in E. coli. Samples of the marker proteins (lane 1) and of UehA (lane 2) were electrophoretically separated on 12.5\% SDSpolyacrylamide gel, and the proteins were stained with Coomassie brilliant blue. (b) Determination of the apparent molecular mass of the purified UehA protein by gel-filtration chromatography on a Superdex $7510 / 300$ column. The protein molecular weight standards used to calibrate the gel-filtration column are indicated by arrows (cytochrome $C=12.4 \mathrm{kDa}$; carbonic anhydrase $=29 \mathrm{kDa}$; serum albumin $=66 \mathrm{kDa}$ ). The UehA protein eluted from the gel-filtration column with the calculated molecular mass of $36 \mathrm{kDa}$. 
with those of the homologous $H$. elongata TeaA protein, whose binding properties were analyzed by isothermal titration calorimetry $\left(K_{\mathrm{d}}\right.$ of $0.19 \pm 0.02 \mu \mathrm{M}$ for ectoine and that of $3.8 \pm 0.07 \mu \mathrm{M}$ for 5hydroxyectoine). ${ }^{15}$ Furthermore, the binding affinities of UehA resemble those of the SBP EhuB of the $\mathrm{ABC}$ transporter EhuABC from $S$. meliloti $\left(K_{\mathrm{d}}\right.$ of $1.6 \pm 0.3 \mu \mathrm{M}$ for ectoine and that of $0.5 \pm 0.1 \mu \mathrm{M}$ for 5 hydroxyectoine) ${ }^{24}$ The affinities for both ligands are basically identical in UehA and EhuB, but TeaA apparently binds ectoine with a 20-fold higher affinity than 5-hydroxyectoine. ${ }^{15}$

\section{Overall structure of the UehA-ectoine crystal complex}

A native dataset of the UehA-ectoine complex was collected at beamline ID-23 (ESRF, Grenoble, France) and scaled using XDS. ${ }^{33}$ Initial phases were obtained by molecular replacement using the program PHASER ${ }^{34}$ with the crystal structure of $H$. elongata ectoine-binding protein TeaA as a template [Protein Data Bank (PDB) entry 2vpn]. ${ }^{15}$ The final structure was refined to a resolution of $2.9 \AA$ A. Data and refinement statistics and model content are summarized in Table 1. The overall structure of UehA in complex with ectoine is depicted in Fig. 4a. As expected for a TRAP-T binding protein, UehA is composed of two domains, which are shown in blue (domain I, residues 1-121 and 212-243) and light brown (domain II, residues 122-211 and 244-310), respectively, in the figure. Each of these domains is composed of a central antiparallel five-stranded $\beta$-sheet. Furthermore, domain I contains six $\alpha$-helices, while domain II harbors eight $\alpha$-helices and one additional $\beta$-strand (Fig. 4a and b), which flank the central sheet on both sites. Interestingly, one strand forms the central $\beta$ sheets of the two domains, thereby crossing the whole protein.

The remarkable features of the UehA structure are reflected in the topology diagram of UehA (Fig. 4b). Both domains contain the mentioned five-stranded
Table 1. Crystallographic parameters

t1.2

Crystal parameters at $100 \mathrm{~K}$

Space group

Unit cell parameters

$a, b, c(\AA)$

$\beta\left(^{\circ}\right)$

Data collection and processing

Wavelength $(\AA)$

Resolution (A)

Mean redundancy

Unique reflections

Completeness (\%)

$I / \sigma$

$R_{\text {sym }}$

Refinement statistics

$R_{F}(\%)$

$R_{\text {free }}(\%)$

rmsd from ideal

Bond lengths $(\AA)$

Bond angles (deg.)

Average $B$-factors $\left(\AA^{2}\right)$

Ramachandran plot

Most favored (\%)

Allowed (\%)

Generously allowed (\%)

Disallowed (\%)

Model content

Monomers/ASU

Protein residues

Ligand

Other

$\begin{array}{rr}P 2_{1} & \mathrm{t} 1.3 \\ & \mathrm{t} 1.4 \\ & \mathrm{t} 1.5\end{array}$

$63.0,61.3,86.3+1.6$

$102.6 \quad \mathrm{t} 1.7$$$
\text { t1.8 }
$$

$\mathrm{t} 1.9$

$0.87260 \quad \mathrm{t} 1.10$

30-2.9 (2.97-2.9) t1.11

$4.0(3.7) \quad \mathrm{t} 1.12$

$14,412 \quad \mathrm{t} 1.13$

99.6 (99.7) t1.14

$11.3(3.6) \quad \mathrm{t} 1.15$

$11.0(42.9) \quad \mathrm{t} 1.16$

$\mathrm{t} 1.17$

t1.18

t1.19

$\mathrm{t} 1.20$

$\mathrm{t} 1.21$

$\mathrm{t} 1.22$

t1.23

$\mathrm{t} 1.24$

$\mathrm{t} 1.25$

$\mathrm{t} 1.26$

t1.27

t1.28

t1.29

t1.30

t1.31

t1.32

$\mathrm{t} 1.33$

$\mathrm{t} 1.34$ $\mathrm{t} 1.35$

Crystal parameters and data collection statistics were derived from XDS. Refinement statistics were obtained from REFMAC5, and Ramachandran analysis was performed using PROCHECK. t1.36 ${ }^{\mathrm{a}} R_{\mathrm{sym}}=\frac{\sum_{h k l} \sum_{i}\left|I_{i}(h k l)-\langle I(h k l)\rangle\right|}{\sum_{h k l} \sum_{i} I_{i}(h k l)}$.

${ }^{\mathrm{b}} R_{F}=\sum_{h k l}|| F_{\mathrm{o}}|-| F_{\mathrm{c}}|| / \sum_{h k l}\left|F_{\mathrm{o}}\right| . R_{\text {free }}$ is calculated as $R_{F}$ but for $5 \%$ randomly chosen reflections that were omitted from all refinement steps.

antiparallel $\beta$-sheets, which have the orders BACJD 367 and GFHEI, respectively. This strand order is found 368 in all other ESR proteins of TRAP-Ts and in class II 369 ABC-dependent SBPs. ${ }^{35}$ However, not only the 370 strand order but also the additional $\beta$-strand and 371 (a)

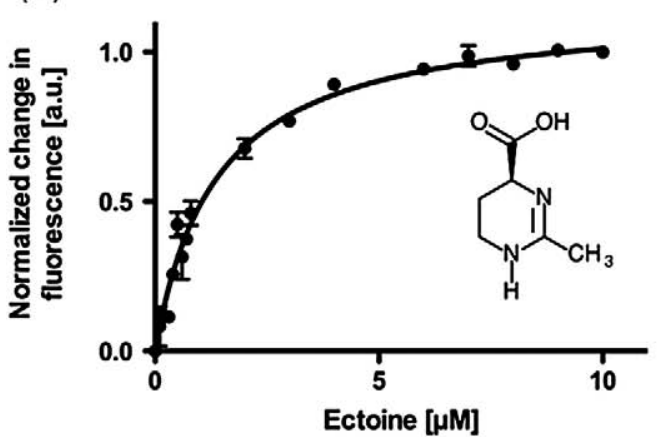

(b)

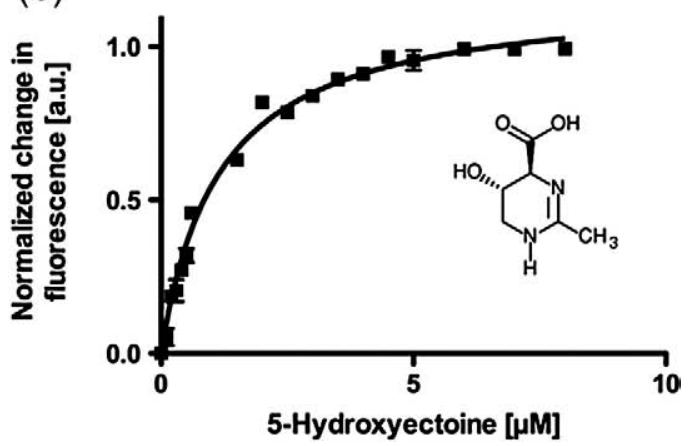

Fig. 3. Dissociation constant of the UehA-ectoine complex. Equilibrium binding titration experiments with ectoine (a) or 5-hydroxyectoine (b) to purified UehA were performed using an intrinsic tryptophan fluorescence-based binding assay. The chemical structures of ectoine and 5-hydroxyectoine are shown in the inset. Equilibrium binding assays were performed in triplicate, and data were analyzed as outlined in Materials and Methods. 


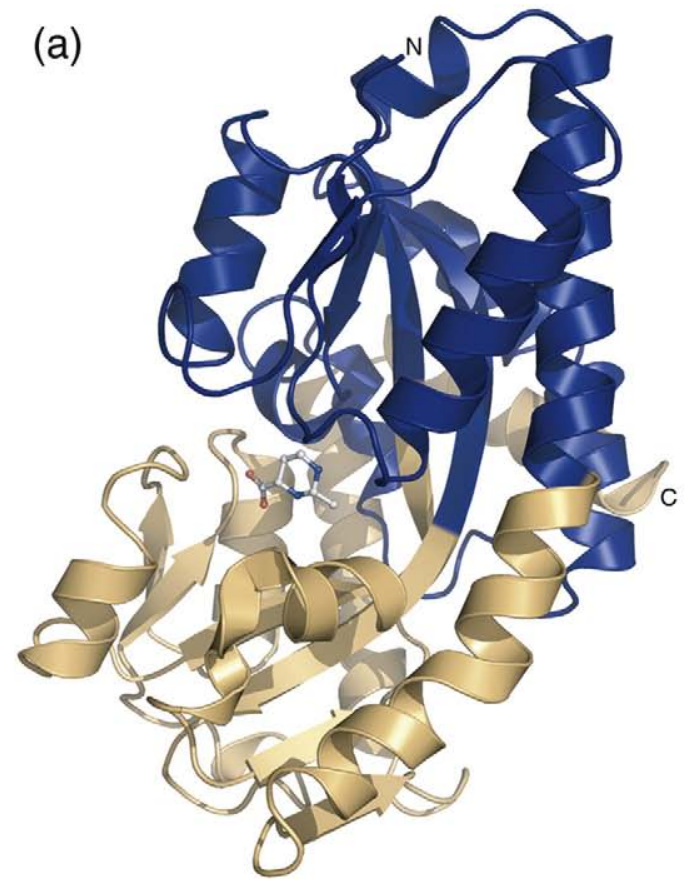

(b)

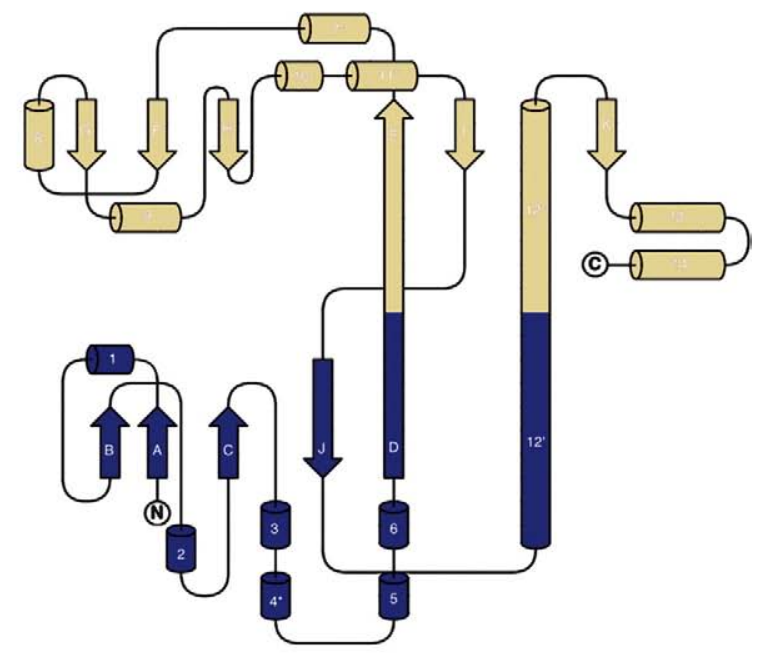

Fig. 4. Overall structure of UehA in complex with ectoine. (a) Domain I of UehA is shown in blue, and domain II of UehA is shown in light orange. The ectoine ligand is shown in ball-and-stick representation. The $\mathrm{N}$ - and C-termini are labeled. (b) A topology diagram of UehA where domain I is in blue and domain II is in dark orange. Helices are labeled by numbers; $\beta$-strands, by letters. $3_{10}$-Helices are indicated by an asterisk.

the number and positioning of the flanking $\alpha$-helices are conserved in all TRAP-dependent ESRs structurally characterized so far. Quite striking is the long helix (residues 225-260) of UehA lying on top of the protein spanning both domains of UehA. Such a long helix is found in all crystal structures of ESR proteins reported so far from TRAP-Ts, although in some structures this helix is interrupted by a kink. ${ }^{16}$

\section{The ectoine ligand-binding site in UehA}

The ligand ectoine resides in a deep cleft located between both domains of UehA (Fig. 4a). Despite the modest resolution of the UehA structure $(2.9 \AA)$, the orientation of ectoine within the ligand-binding site could be determined unambiguously as demonstrated by a $1 F_{\mathrm{o}}-F_{\mathrm{c}}$ omit map contoured at $3 \sigma$ (Fig. 5). Residues from both domains (shown in blue and yellow in Fig. 5) of UehA form the ectoine-binding site: one ligandinteracting residue is provided by domain I, and five ligand-interacting residues originate from domain II. Hence, residues from both domains of UehA stabilize and orient the ligand within the binding pocket, a general feature found in ESRs and SBPs. Three salt bridges contribute to the stabilization and positioning of ectoine within the UehA ligand-binding pocket: the carboxyl moiety of ectoine interacts with Arg144, the imido moiety of ectoine interacts with Glu9 and, furthermore, the imido and carboxylate moieties of ectoine both interact with Asn184. In addition, cation-pi inter- actions of the delocalized positive charge of ectoine 402 are formed by Trp167, Phe188 and Phe209 of UehA 403 and make important contributions to ligand bind- 404 ing. Hydrophobic interactions of ectoine with 405 Glu8, Phe66, Met146 and Phe187 provide further 406 stabilization of the ligand (Fig. 5).

Compatible solutes are usually excluded from the 408 immediate hydration shell of proteins due to unfa- 409 vorable interactions with the protein backbone. ${ }^{36}{ }_{410}$ However, in ESRs and SBPs, high-affinity interac- 411 tions with the ligand $\left(K_{\mathrm{d}}\right.$ values in the low 412 micromolar or even nanomolar range) are required 413 to permit the efficient scavenging of compatible 414 solutes from scarce environmental sources. The 415 crystal structures of several compatible solute bind- 416 ing proteins have recently been determined in 417 complex with their specific ligands. ${ }^{24,31,32,37-39}$ A 418 combination of direct (salt bridges and hydrogen 419 bonds) and non-direct (cation-pi) interactions has 420 been observed at a structural level not only in the 421 ectoine-specific ESRs UehA and TeaA but also in 422 ABC-dependent SBPs with specificity for compatible 423 solutes, such as glycine betaine, proline betaine and 424 dimethylsulfonioacetate. Examples include the 425 ectoine/5-hydroxyectoine-binding protein EhuB 426 from S. meliloti, ${ }^{24}$ the glycine betaine-proline 427 betaine-dimethylsulfonioacetate binding protein 428 OpuAC from Bacillus subtilis, ${ }^{31,37}$ the choline-acet- 429 ylcholine binding protein ChoX from S. meliloti ${ }^{32}$ and 430 the glycine betaine-proline betaine binding protein 431 ProX from E. coli and that from Archaeoglobus 432 fulgidus. ${ }^{38,39}$ Hence, in SBP and ESR originating 433 


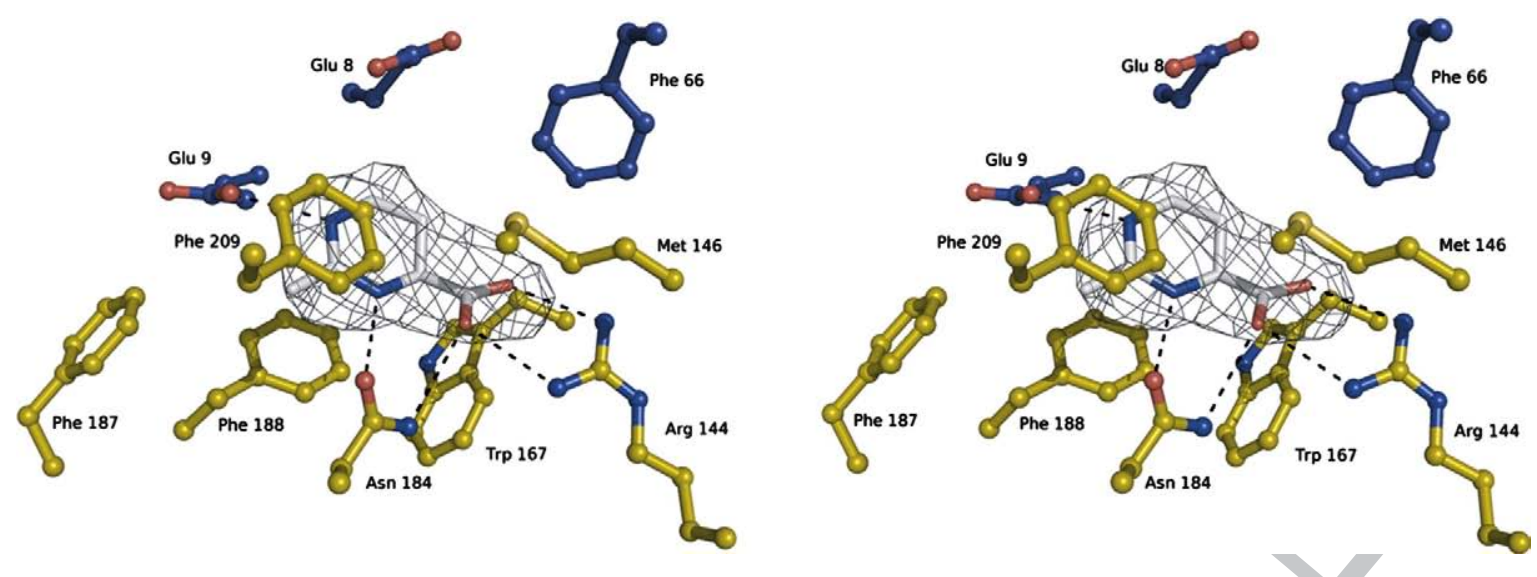

Fig. 5. The ectoine ligand-binding site in UehA. Residues of domain I of UehA participating in ligand binding are shown in blue and residues originating from domain II of UehA are shown in orange and the numbers of the amino acids forming the ligand-binding pocket are labeled. The ectoine ligand is shown in ball-and-stick representation. Hydrogen bonds and salt bridges are highlighted by black dashed lines. The gray mesh indicates a $1 F_{o}-F_{c}$ omit electron density map of the ectoine ligand-binding site contoured at $3 \sigma$. For electron density map calculations, the ligand was omitted from the refinement.

from a diverse range of microbial species, common types of molecular interactions are employed to efficiently and specifically bind compatible solutes within binding pockets.

\section{Comparison of the UehA structure with the ectoine-binding ESR TeaA}

The ESR UehA from S. pomeroyi DSS-3 and TeaA from $H$. elongata are each part of an ectoine/5hydroxyectoine-specific TRAP-T system. However, their physiological functions differ: TeaABC is involved in the acquisition of ectoine/5-hydroxyectoine as osmoprotectants from environmental sources, ${ }^{23}$ whereas the UehABC system serves as an ectoine/5-hydroxyectoine transporter for nutritional purposes (Fig 1b). Several uptake systems for compatible solutes, such as the $\mathrm{ABC}$ transporter OpuA from Lactococcus lactis, ${ }^{40}$ the BCCT-type transporter BetP from Corynebacterium glutamicum ${ }^{41}$ and the MFS-type transporter ProP from E. coli, ${ }^{42}$ are regulated at the level of transport activity by high osmolarity. These transport systems respond to a sudden osmotic up-shock with increased import of compatible solutes to protect the microbial cells from the detrimental effects of high osmolarity. Such a response via the UehABC system is quite unlikely because both ectoine and 5-hydroxyectoine simply do not serve as osmoprotectants for S. pomeroyi DSS3. These tetrahydropyrimidines, in contrast to the metabolically inert osmoprotectant glycine betaine, only serve as nutrients in S. pomeroyi DSS-3 (J.B. and E.B., unpublished results); consequently, there is no physiological need for the cell to regulate ectoine and 5-hydroxyectoine uptake in response to increased osmolarity of the environment.

Despite these physiologically different functions, the TeaA and UehA proteins are closely related in amino acid sequence (62\% amino acid sequence identity and $82 \%$ homology), and this close relatedness is reflected in the crystal structures of both proteins. A structural superposition of UehA and 473 TeaA reveals an rmsd of $0.8 \AA$ over $309 \mathrm{C}^{\alpha}$ atoms 474 (data not shown). Besides the overall fold, the 475 architectural arrangements of the residues interact- 476 ing with the ectoine ligand are also identical in 477 UehA (Fig. 5) and TeaA (data not shown). The 478 crystal structure of the TeaA-ectoine complex was 479 refined by Kuhlmann et al. to a resolution of $1.55 \AA \AA^{15}{ }_{480}$ This high resolution allowed the conclusion that 481 residues Glu44 and Ser 45 of TeaA interact indirectly 482 with the ectoine ligand via a bridging water 483 molecule. Since the UehA structure reported here 484 was determined only at modest resolution $(2.9 \AA)$, 485 we refrained from including water models in our 486 final model. However, given the close amino acid 487 sequence and structural relatedness of UehA and 488 TeaA, it seems reasonable to assume that a bridging 489 water molecule might also mediate similar interac- 490 tions of Glu44 and Ser45 in UehA with the ectoine 491 ligand. Remarkably, despite the different physiolo- 492 gical tasks performed by the TeaABC and UehABC 493 transporters, nature has chosen the same architec- 494 ture of the substrate-binding site in TeaA and UehA 495 to effectively capture the ligand ectoine.

The structure of TeaA revealed a pronounced 497 negatively charged surface. However, the moderate 498 halophile H. elongata can grow over a wide range of 499 salinities (up to $10 \% \mathrm{NaCl}$ ). Such an exposure of 500 negative charges to the surface is common among 501 proteins of extremely halophilic archaea. ${ }^{15}$ Interest- 502 ingly, the surface of UehA also contains mainly 503 negatively charged amino acids, although S. pomer- 504 oyi DSS-3 lives in a marine habitat, an environment 505 of moderately elevated salinity (approximately 3.5\% 506 $\mathrm{NaCl}$ ) (data not shown).

\section{Comparison of the ESR UehA with the ectoine-binding SBP EhuB}

The EhuB protein is part of an $\mathrm{ABC}$ transport 510 system that serves for the uptake of ectoine and 5- 511 
(a)
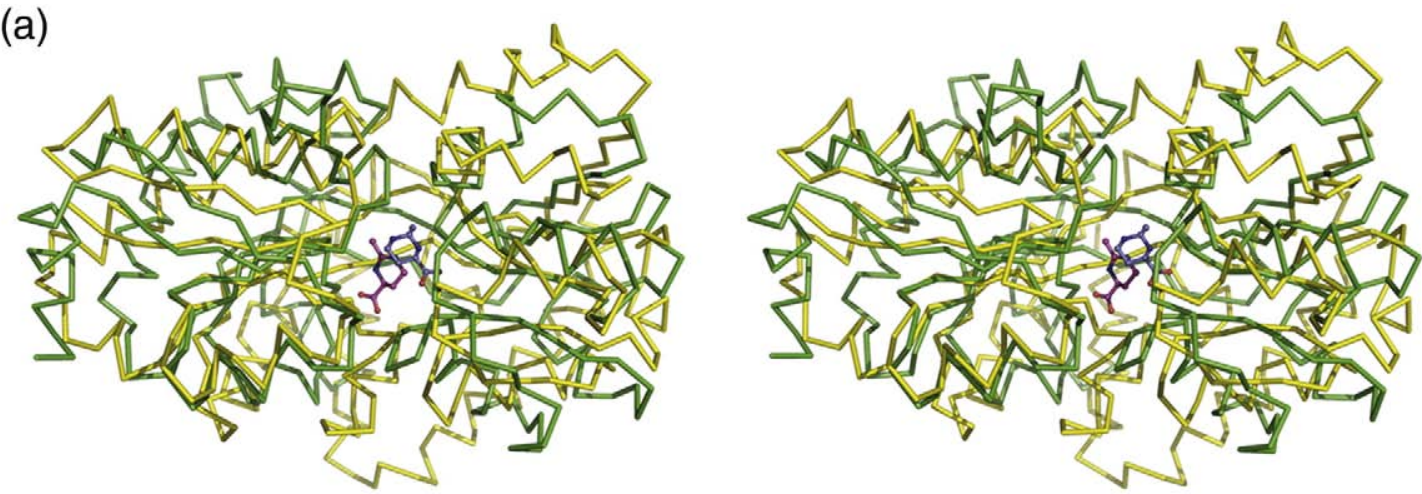

(b)
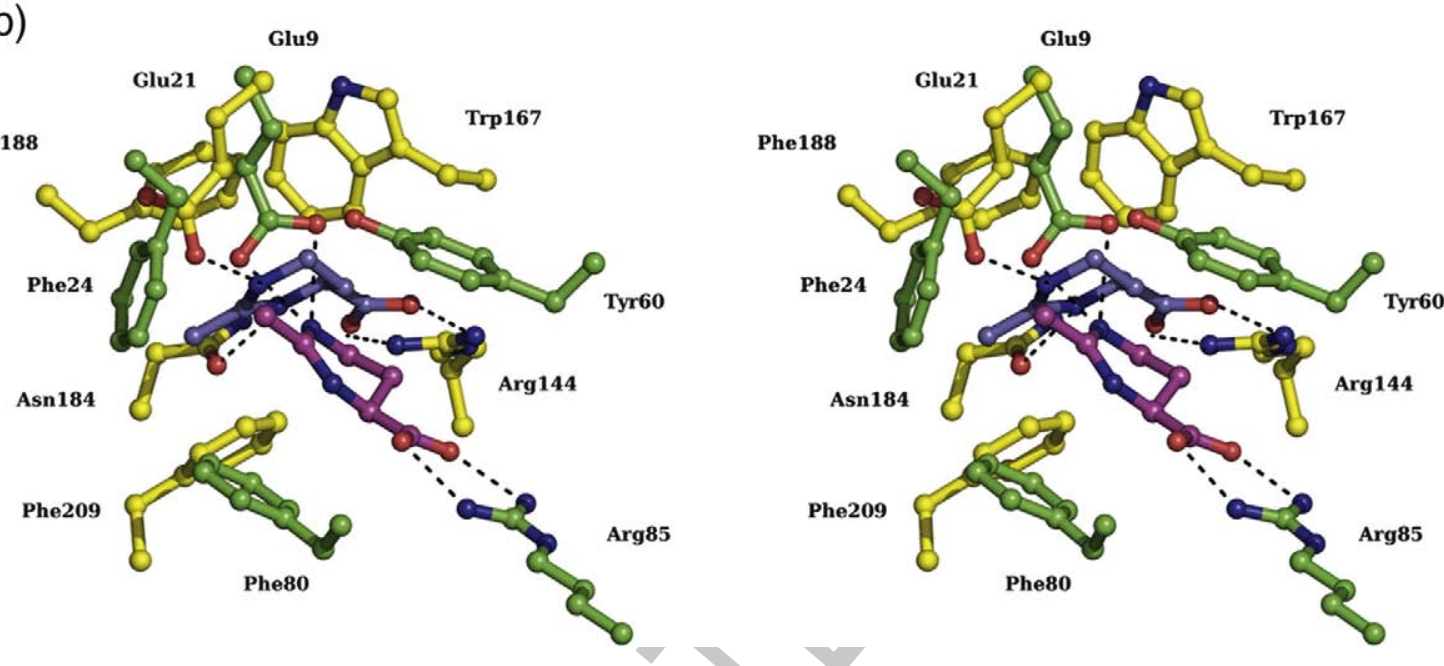

Fig. 6. Structural superposition of the EhuB and UehA ectoine-binding proteins. (a) Stereo view of a structural superposition of the SBP EhuB (green) from S. meliloti and the ESR UehA from S. pomeroyi (yellow). The ectoine ligand is shown in ball-and-stick representation in either magenta for EhuB or dark cyan for UehA. (b) Detailed view of the ectoine ligand-binding site in EhuB and UehA. The overall structures of EhuB and UehA were used as a reference point for the comparison of the ectoine ligand-binding sites. Residues that directly interact with ectoine and the ligands are shown in ball-and-stick representation. Hydrogen bonds and salt bridges between the ligand and the protein are highlighted by black dashed lines. The color coding for residues originating from either EhuB or UehA is identical with that shown in (a).

hydroxyectoine for nutritional purposes in the soil bacterium S. meliloti. ${ }^{29}$ The crystal structure of EhuB was solved in complex with either ectoine or 5hydroxyectoine. ${ }^{24}$ In their recent structural analysis of TeaA, Kuhlmann et at. ${ }^{15}$ compared TeaA and EhuB by superimposing the common ligand ectoine. Applying this strategy, they detected significant differences in the architecture of the ligand-binding site and mode of ligand binding between the EhuB and TeaA proteins. However, when we followed this approach by using the ectoine ligand bound by 522 UehA as the anchor point for the EhuB-UehA 523 comparison, we found a clear misalignment of the 524 protein backbone of both proteins (data not shown). 525 Therefore, we used the protein structure of UehA 526 and EhuB for an overall superposition (Fig. 6a). This 527 resulted in an rmsd of $3.0 \AA$ for $162 \mathrm{C}^{\alpha}$ atoms (Fig. 528 $6 a)$. In this superposition, it is evident that the 529 central antiparallel $\beta$-sheets of both domains align 530 well, while the long helix 12 (Fig. 4b) and the helices 531

Fig. 7. Structural superposition of TRAP-derived SBPs. (a) Ribbon representations of a structural superimposition of UehA (orange) and SiaP (yellow). The ligands ectoine in UehA and sialic acid in SiaP are shown in ball-and-stick representation. (b) Detailed views of the structure of the ligand-binding sites present in UehA (orange) and SiaP (yellow). Residues forming the ligand-binding site and the ligands are shown in ball-and-stick representation. Helix 3 is displayed in cartoon representation. Residues lining the binding side are labeled. Here, the first residue always refers to UehA; the second, to the structurally corresponding residue of SiaP. If no corresponding residue was present, only a single residue is indicated. (c) The structures of UehA (orange) and TakP (cyan) are superimposed and represented as ribbons. The ligands ectoine (UehA) and pyruvate (TakP) are shown in ball-and-stick representation. (d) Detailed views of the ligand-binding site of UehA (orange) and TakP (cyan) are presented. Residues forming the ligand-binding site and the ligands are shown in ball-and-stick representation. Helix 3 is displayed in cartoon representation. Residues lining the binding side are labeled. Here, the first residue always refers to UehA; the second, to the structurally corresponding residue of TakP. If no corresponding residue was present, only a single residue is indicated. 
(a)
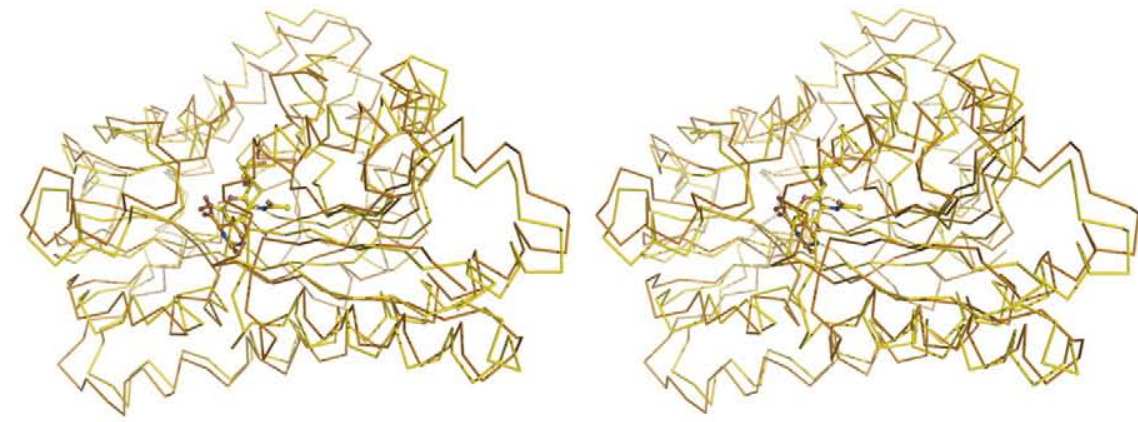

(b)
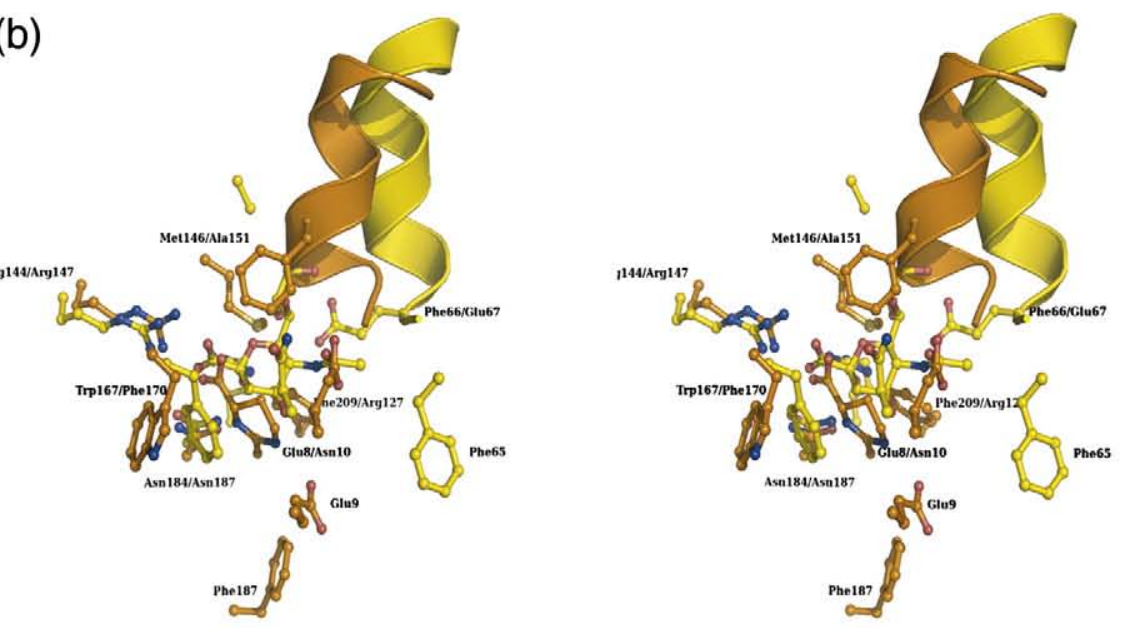

(c)
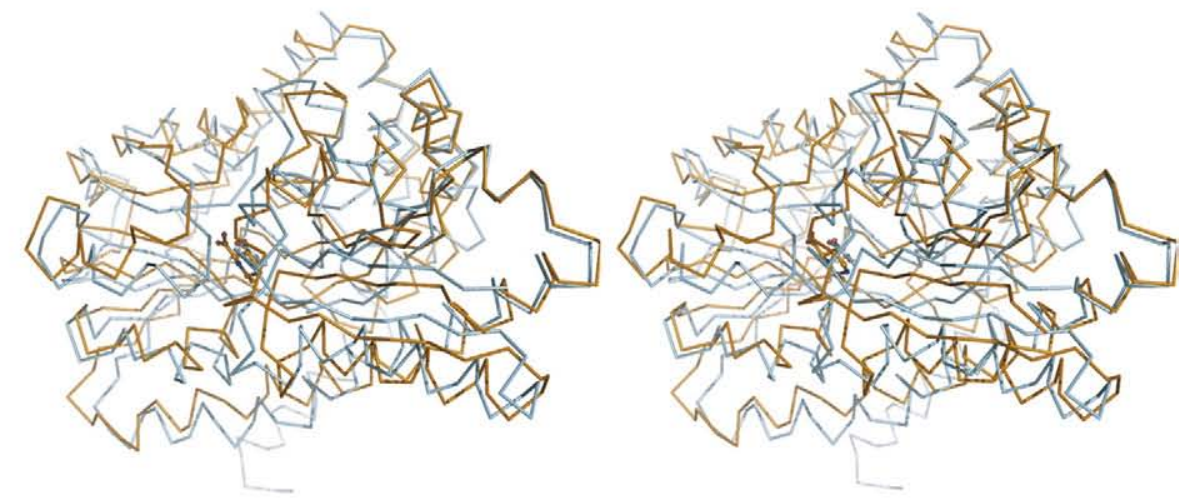

(d)

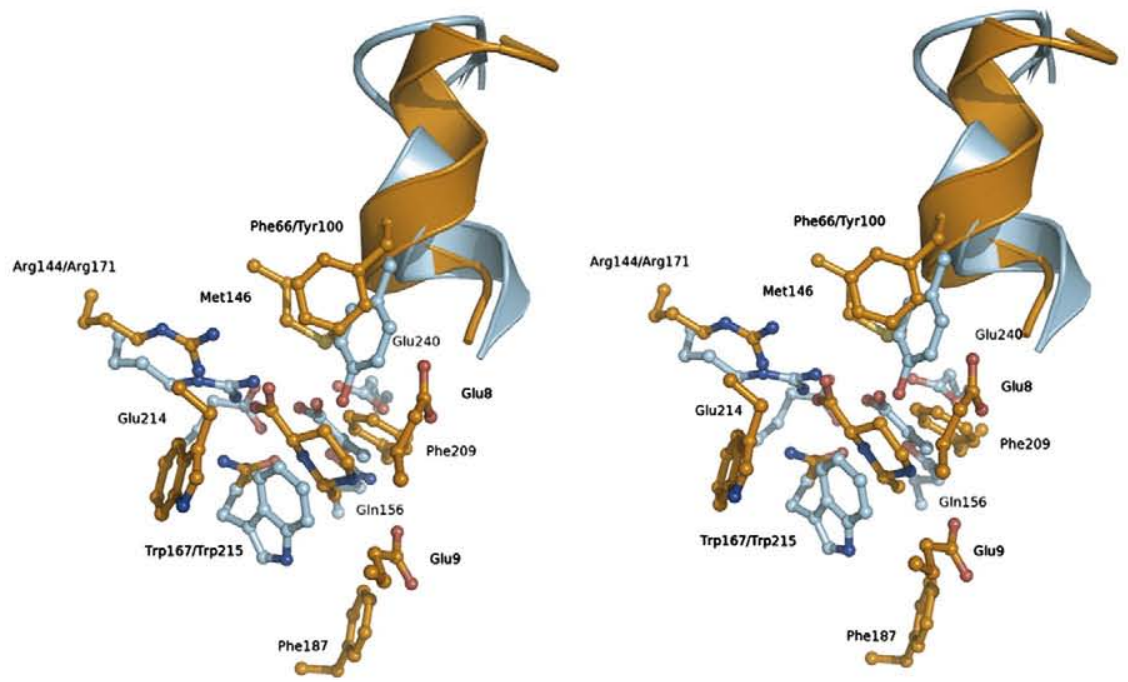

Fig. 7 (legend on previous page) 
of the C-terminal part of UehA (Fig. 6a) have no counterpart in EhuB. The alignment of the core part of both proteins underlines the structural similarities of SBPs from ABC transporters and ESRs from TRAP-Ts. It has already been suggested that both types of ligand-binding proteins evolved from a common ancestor and perform similar functions in ABC- and TRAP-type transporters. 8,17

Figure $6 \mathrm{~b}$ represents a detailed view of the aligned ectoine-binding site present in UehA (yellow) and in EhuB (green). The ligands are displayed in ball-andstick representation in cyan for UehA and in magenta for EhuB. In our alignment approach, which used the protein backbones as anchor points, a detailed view of the binding site clearly demonstrates that the positions of the ectoines are different in UehA and EhuB. Both a rotational shift and a translational shift would be required to adequately superimpose the ligands. Despite these shifts, the ligand-protein interactions in UehA and EhuB are identical. The carboxylate of ectoine interacts with an arginine residue (Arg144 in UehA and Arg85 in EhuB), and the imido moiety interacts with a glutamate (Glu9 in UehA and Glu21 in EhuB). Additionally, a hydrogen bond is present between Asn184 and the imido moiety of ectoine in UehA, but there is no counterpart for this type of interaction in EhuB. These protein-ligand contacts are completed by cation-pi interaction between the delocalized positive charges of the ectoine ligands and aromatic residues present in the two binding proteins. However, these aromatic residues in UehA and EhuB are swapped between the domains: they originate from domain I in EhuB and from domain II in UehA. Another interesting swap occurred for the arginine residue that interacts with the carboxylate moiety of ectoine. Arg144 of UehA is structurally conserved in all ESRs of TRAP-Ts that were structurally characterized so far. ${ }^{12-16}$ The same holds true for Arg85 of EhuB, which is equally conserved in binding proteins from $\mathrm{ABC}$ transporters likely being involved in ectoine uptake. ${ }^{24}$ However, as in the case of the aromatic residues participating in cation-pi interactions, the two arginines stem from different domains: domain I in EhuB and domain II in UehA. In both the UehA and EhuB structures, the ligand is completely shielded from the solvent and buried deep within the protein. Thus, both proteins employ the same principles of ligand binding and generate an overall architecture to perfectly accommodate ectoine, but the detailed arrangements of the amino acid residues forming the ligand-binding site are different in UehA and EhuB.

\section{Structural comparison of UehA with ESRs of other TRAP-Ts}

Recently, the crystal structures of seven TRAPassociated ESRs have been characterized by X-ray crystallography. A comparison of each of these structures with UehA (Fig. 7) revealed that all TRAP-dependent ESRs analyzed by X-ray crystal- lography adopt the same overall tertiary structure. 593 Not only are the topological arrangements of the 594 central $\beta$-sheets identical but also the number of the 595 flanking helices and their structural arrangement are 596 virtually superimposable. This also includes the 597 extended helix (helix 12 in UehA; see Fig. 4a) 598 spanning in each case the whole ESR structure. $\quad 599$

As an example of this remarkable structural 600 similarity between the ESRs of TRAP systems with 601 different substrate specificities, structural align- 602 ments of UehA with the N-acetyl-5-neuramic acid- 603 specific binding protein $\mathrm{SiaP}^{12,17}$ and the $\alpha$-keto 604 acid-specific binding protein TakP ${ }^{13}$ are shown in 605 Fig. 7. The overlay of UehA (orange) and SiaP 606 (yellow) is provided in Fig. 7a. Both proteins align 607 with an rmsd of $2.1 \AA$ over $310 \mathrm{C}^{\alpha}$ atoms, despite the 608 fact that the amino acid sequence homology 609 between UehA and SiaP is only 26\%. Another 610 example is provided in Fig. 7c. Here, UehA and 611 TakP were superimposed, resulting in an rmsd of 612 $2.4 \AA$ over $310 \mathrm{C}^{\alpha}$ atoms (the amino acid sequence 613 identity between these two proteins is only $18 \%$ ). 614 Structural alignments of UehA with other ESRs 615 revealed similar values, indicating that the overall 616 structure of all TRAP-dependent ESRs is, within 617 experimental error, identical, and this includes the 618 number and orientation of the flanking helices in 619 these binding proteins as well.

620

The common structural architecture of TRAP- 621 associated ESRs implies that these SBPs might all 622 interact in the same way with the membrane 623 components of TRAP-type transport systems. How- 624 ever, significant functional differences seem to exist 625 with respect to the oligomeric composition of the 626 various ESRs in solution and in crystals. Both the 627 TakP protein from R. sphaeroides $^{13}$ and the ESR from 628 T. maritima ${ }^{16}$ form dimers in solution. Elaborate 629 models have been proposed to explain how such a 630 dimeric complex of the ESR might channel the 631 ligand to the membrane components of the TRAP 632 system for solute import. The other ESRs, for which 633 structural information is available, are all monomers 634 in solution, and it is thus likely that these ligand- 635 binding proteins also interact as monomers with the 636 cognate membrane components of the various 637 TRAP-Ts. UehA belongs to this latter group since 638 it elutes as a monomer from a size-exclusion column 639 (Fig. 2b). A monomeric SBP is also observed in the 640 crystal structures of three microbial ABC importers 641 in complex with their cognate ligand-binding 642 proteins. In these structures, a monomeric binding 643 protein interacts simultaneously with both trans- 644 membrane components of the ABC transporter. ${ }^{4-45} 645$

The structural relatedness of the various ESRs 646 from TRAP-Ts raises the question of how substrate 647 specificity for the ligand is generated within this 648 protein family. Detailed structural comparisons of 649 the ligand-binding sites of UehA-SiaP and UehA- 650 TakP are provided in Fig. $7 \mathrm{~b}$ and Fig. $7 \mathrm{~d}$, respec- 651 tively. Residues of both proteins that interact with 652 the respective ligand are shown in ball-and-stick 653 representation (orange color coding for UehA and 654 yellow color coding for SiaP in Fig. 7b), and the 655 
corresponding ligands (ectoine in UehA and $\mathrm{N}$ acetyl-5-neuramic acid in SiaP) are shown in balland-stick representation in orange and yellow, respectively.

The well-conserved arginine residues (Arg144 in UehA and Arg147 in SiaP) perfectly align in this structural comparison. Other residues such as Asn184 (UehA) and Asn187 (SiaP) or Trp167 (UehA) and Phe170 (SiaP) also correspond, whereas certain residues such as Glu9 (UehA) are specific for the particular ESR. These specific interactions are likely a consequence of the chemical structure of the ligand that has to be complexed by a given ESR. However, most striking in this comparison is the positioning of helix 3 in the various ESRs. The exact positioning of this helix in UehA prohibits binding of sialic acid (a substrate for SiaP) because of a steric clash between the side chain of the ligand and the backbone of the helix. Furthermore, in both the UehA and SiaP proteins, one residue of helix 3 participates in ligand binding: these are Phe66 in UehA and Glu67 in SiaP. Thus, the exact position of helix 3 is a critical determinant for the size of the ligand-binding pocket, thereby significantly contributing to the precise positioning of the individual ligands within the binding site.

A similar situation is observed when one compares the ligand-binding sites of UehA (orange) with those of TakP (cyan; Fig. 7d), an ESR that is specific for $\alpha$-keto acids. Again, the two conserved arginine residues align fairly well (Arg144 in UehA and Arg171 in TakP) and in both proteins two residues originating from helix 3 (Phe66 in UehA and Tyr100 in TakP) interact with the ligand. Since ectoine and pyruvate are of similar size, the position of helix 3 is comparable in both proteins and the displacement of this helix observed by comparing the UehA and SiaP structures is less pronounced. Nevertheless, in all structures analyzed, helix 3 adopts a specific and unique position within the family of TRAPassociated ESRs. Our analysis of the available structures of these types of proteins strongly suggests that helix 3 acts as a "selectivity helix" by modulating the volume available for a given ligand within the binding pocket and that helix 3 provides at least one residue that directly interacts with the given ligand.

\section{UehA as a structural and functional template for other putative ectoine-binding proteins from TRAP transport systems}

A BLAST search ${ }^{46}$ for proteins from both bacteria and archaea that are related to the amino acid sequence of the S. pomeroyi DSS-3 UehA protein was conducted using the DOE Joint Genome Institute Web servert. Proteins that are similar to UehA were readily detected, and the top 10 UehA-related proteins were retained for further analysis and their amino acid sequences were aligned using the ClustalW algorithm ${ }^{47}$ (see Supplementary Fig. 1).

thttp:/ / www.jgi.doe.gov/
Each of these proteins originates from Gram- 714 negative bacteria and contains an N-terminal signal 715 sequence that directs it via the Sec pathway into the 716 periplasmic space. The 10 UehA-related proteins are 717 each encoded as part of a gene cluster (dctPQM) 718 whose products are annotated as DctPQM-type 719 TRAP transport systems. They all stem from 720 microorganisms belonging to the proteobacteria, 721 and all live in either marine or saline habitats. These 722 microorganisms comprise Roseobacter spp. (two 723 species), Marinobacter spp. (three species) and 724 species from the Oceanibulbus, Oceanicola, Auranti- 725 monas, Fulvimarina, Halomonas and Reinekea genera. 726

These ESR proteins all align with the UehA 727 protein from S. pomeroyi DSS-3 without any gaps, 728 comprise a similar number of amino acids and 729 exhibit a degree of amino acid sequence identity 730 with UehA that ranges from $74 \%$ to $96 \%$ (see 731 Supplementary Fig. 1). We then inspected the 732 aligned UehA-related proteins for the conservation 733 of those nine amino acid residues that form the 734 ectoine-binding pocket in the UehA structure (Fig. 735 5). Eight of the ligand-interacting residues present in 736 UehA are fully conserved. A minor variation occurs 737 at a position that corresponds to Phe188 in UehA; at 738 this site, the Phe residue is conservatively replaced 739 by either Tyr or Trp residues in some proteins. The 740 strict conservation of the ligand-interacting residues 741 from UehA in the compiled and aligned proteins 742 strongly suggests that each of these ESRs is actually 743 an ectoine-binding protein with an overall structure 744 resembling that of the S. pomeroyi DSS-3 UehA 745 protein (Fig. 3a).

746

This suggestion is strengthened by the finding that 747 not only is the ligand-binding protein conserved but 748 also the small and large integral membrane compo- 749 nents of the different TRAP transport systems are 750 related to the corresponding proteins (TeaB-UehB 751 and $\mathrm{TeaC}-\mathrm{UehC}$ ) from the H. elongata and S. 752 pomeroyi DSS-3 ectoine/5-hydroxyectoine TRAP 753 uptake systems. The amino acid sequence identity 754 of the UehB-related proteins ranges from $48 \%$ to 755 $98 \%$, whereas the amino acid sequence identity of 756 those related to UehC ranges from $71 \%$ to $96 \%$. The 757 S. pomeroyi DSS-3 uehABC gene cluster is also 758 followed by a universal stress protein (Usp) usp 759 gene (Fig. 1a). Genes encoding homologs of these 760 Usps $^{48}$ have been observed to be adjacent to the 761 genes encoding the components of many TRAP-Ts, 762 but their role in connection with TRAP-type 763 transporters has not yet been explored. ${ }^{8}$

\section{Conclusions}

Uptake of ectoine and 5-hydroxyectoine in S. 766 pomeroyi DSS is mediated by the substrate-inducible 767 TRAP-T UehABC. In contrast to TeaABC from H. 768 elongata, the only other functionally characterized 769 TRAP-T for ectoine and 5-hydroxyectoine, the 770 UehABC system is not involved in osmoprotection 771 and instead imports both ectoine and 5-hydroxyec- 772 toine for nutritional purposes. The ESR of the 773 
UehABC system, UehA, binds both ectoine and 5hydroxyectoine with high specificity and affinity. The structure of UehA in complex with ectoine revealed the molecular determinants for ligand binding. Databank searches identified a group of UehA-related putative ectoine-binding proteins from marine microorganisms in which the residues forming the ligand-binding pocket are strictly conserved, implying that the structure of UehA can serve as template for functional studies of other ectoine-specific ESRs. The ligand-binding pocket of UehA is superimposable to that of the ectoinebinding protein TeaA, and the types of interactions between residues present in UehA-TeaA and its ligand ectoine can also be found in the ectoinespecific SBP EhuB from S. meliloti.

We compared the crystal structure of UehA with the structures of seven ESRs originating from TRAP systems with different substrate specificities. The overall fold of all ESRs is virtually identical, and we found that the size of the substrate-binding pocket in these proteins is dictated by only one helix (helix 3 ). The positioning of this helix modulates the volume of the binding pocket with the ESR and apparently aids in determining the selectivity of ligand binding.

\section{Materials and Methods}

\section{Chemicals}

Ectoine and 5-hydroxyectoine were obtained from bitop AG (Witten, Germany) and were generous gifts of Dr. T. Schwarz. $\left[{ }^{14} \mathrm{C}\right]$ Ectoine $\left(4.22 \mathrm{MBq} \mathrm{mmol}^{-1}\right)$ was prepared and purified as detailed previously ${ }^{49}$ and was a kind gift of Dr. M. Jebbar (University of Rennes, Rennes, France). Glycine betaine, proline and trehalose were purchased from Sigma-Aldrich (München, Germany). Anhydrotetracycline and desthiobiotin were obtained from IBA (Göttingen, Germany).

\section{Bacterial strains, plasmids and culture conditions}

S. pomeroyi DSS-3 (DSM 15171) ${ }^{20}$ was obtained from the German Collection of Microorganisms and Cell Cultures (DSMZ; Braunschweig, Germany). S. pomeroyi DSS-3 was propagated and maintained on half-strength YTTS complex medium as described previously. ${ }^{50}$ For the growth of $S$. pomeroyi DSS-3 in a chemically defined medium, we used the basal minimal medium described previously ${ }^{51}$ at $30{ }^{\circ} \mathrm{C}$. This medium had the following composition: $200 \mathrm{mM} \mathrm{NaCl}, 50 \mathrm{mM} \mathrm{MgSO}{ }_{4} \times 7 \mathrm{H}_{2} \mathrm{O}, 10 \mathrm{mM} \mathrm{KCl}$, $10 \mathrm{mM} \mathrm{CaCl} \times 2 \mathrm{H}_{2} \mathrm{O}, 190 \mathrm{mM} \mathrm{NH} \mathrm{N}_{4} \mathrm{Cl}, 0.33 \mathrm{mM}$ $\mathrm{K}_{2} \mathrm{HPO}_{4}, 0.1 \mathrm{mM} \mathrm{FeSO} 4 \times 7 \mathrm{H}_{2} \mathrm{O}, 50 \mathrm{mM}$ Mops, $\mathrm{pH}$ 7.5, $28 \mathrm{mM}$ glucose and $5 \mathrm{ml} \mathrm{l}^{-1}$ each of two vitamin solutions. For cells that were grown at elevated salinity, the total concentration of $\mathrm{NaCl}$ in the medium was raised to $0.4 \mathrm{M}$. In cultures of $S$. pomeroyi DSS-3 that used ectoine as the sole carbon source, the glucose content of the basal minimal medium was replaced by $28 \mathrm{mM}$ ectoine. E. coli strain BL21(DE3) (Stratagene, La Jolla, CA) was used for the overexpression of the $S$. pomeroyi DSS-3 uehA gene carried by the expression plasmid $\mathrm{pBJ} 20$, a derivative of the vector pASK-IBA6 (IBA). BL21(pBJ20) strains were propagated on LB-agar plates containing ampicillin
(100 $\mu \mathrm{g} \mathrm{ml}^{-1}$ ). For UehA overproduction, the pBJ20-833 carrying BL21 strain was propagated in a defined minimal 834 medium (MMA) ${ }^{52}$ supplemented with $100 \mu \mathrm{g}$ ampicillin 835 $\mathrm{ml}^{-1}, 0.4 \%(\mathrm{w} / \mathrm{v})$ casamino acids and $0.4 \%(\mathrm{w} / \mathrm{v})$ glucose 836 as the carbon source with additional $0.2 \mathrm{~g}^{-1}$ of 837 $\mathrm{MgSO}_{4} \times 7 \mathrm{H}_{2} \mathrm{O}$ and $1 \mathrm{mg} \mathrm{l}^{-1}$ of thiamine. 838

Uptake assays for [ $\left[{ }^{\mathbf{1 4}} \mathbf{C}\right]$ ectoine by $\mathbf{S}$. pomeroyi 839 DSS-3 cells

Uptake assays for $\left[{ }^{14} \mathrm{C}\right.$ ]ectoine $\left(4.22 \mathrm{MBq} \mathrm{mmol}^{-1}\right)$ by $S .841$ pomeroyi DSS-3 cells were conducted as described pre- 842 viously by Bursy et al. ${ }^{28}$ Cells were grown in the basal 843 minimal medium to an $\mathrm{OD}_{578}$ of approximately 1.0.844 Samples of $2 \mathrm{ml}$ were taken, and $\left[{ }^{14} \mathrm{C}\right]$ ectoine was added 845 to the cells at a final concentration of $19 \mu \mathrm{M}$. At the 846 indicated time intervals, $0.3-\mathrm{ml}$ samples were withdrawn 847 and the radiolabeled ectoine taken up by the cells was 848 determined by liquid scintillation counting. Cells grown 849 under the following conditions were used for the $\left[{ }^{14} \mathrm{C}\right] 850$ ectoine uptake experiments: (i) basal minimal medium 851 with glucose as the carbon source, (ii) basal minimal 852 medium with glucose as the carbon source and a total 853 $\mathrm{NaCl}$ content of $0.4 \mathrm{M}$ and (iii) basal minimal medium 854 with ectoine $(28 \mathrm{mM})$ as the carbon source. Prior to the 855 $\left[{ }^{14} \mathrm{C}\right]$ ectoine uptake assays, cells were washed two times in 856 basal minimal medium with glucose $(28 \mathrm{mM})$, except for 857 the cells that were pre-grown in the presence of $0.4 \mathrm{M} 858$ $\mathrm{NaCl}$. For these cells, $0.4 \mathrm{M} \mathrm{NaCl}$ was added to the 859 washing solution to prevent an osmotic down-shock of the 860 cells.

861

Construction of a uehA expression plasmid and overproduction and purification of the recombinant 862 UehA protein

Chromosomal DNA of $S$. pomeroyi DSS-3 was prepared 865 using tip-20 QIAGEN columns (QIAGEN, Hilden, Ger- 866 many). Synthetic oligonucleotides were used to amplify 867 by PCR the uehA coding region from the S. pomeroyi DSS-3 868 genomic DNA without the DNA segment specifying the 869 predicted UehA N-terminal signal sequence. The ampli- 870 fied uehA DNA segment was ligated into the pASK-IBA6 871 expression vector yielding plasmid pBJ20. The uehA 872 coding region was inserted into pASK-IBA6 in-frame 873 with an upstream ompA signal sequence and the codons 874 specifying the Strep-tag II affinity peptide. This allowed 875 the secretion of the Strep-tag II-UehA fusion protein into 876 the periplasmic space of $E$. coli, from which it could be 877 released by cold osmotic shock and recovered by affinity 878 chromatography on Strep-Tactin Sepharose (IBA). The 879 nucleotide sequence of the $u e h A$ coding region present in 880 plasmid pJB20 was verified by DNA sequence analysis, 881 and this was carried out by Eurofins MWG Operon 882 (München, Germany).

883

Six 1-1 minimal media were inoculated with an over- 884 night culture of strain BL21(pBJ20) and were then 885 incubated in an aerial shaker set at $170 \mathrm{rpm}$ at $32{ }^{\circ} \mathrm{C}$. 886 UehA production was initiated by the addition of the 887 inducer of the tet promoter present in $\mathrm{pBJ} 20$, anhydrote- 888 tracycline (final concentration $=0.2 \mu \mathrm{g} \mathrm{ml}^{-1}$ ), at an $\mathrm{OD}_{578} 889$ of 0.7 , and growth of the cultures was then continued for 890 two additional hours. Subsequently, the E. coli cells were 891 harvested by centrifugation $(10 \mathrm{~min}, 6000 \mathrm{~g})$. The cell pellet 892 was resuspended in $50 \mathrm{ml}$ ice-cold $\left(4^{\circ} \mathrm{C}\right)$ buffer P $(100 \mathrm{mM} 893$ Tris- $\mathrm{HCl}$, pH 7.5, $1 \mathrm{mM}$ ethylenediaminetetraacetic acid 894 and $500 \mathrm{mM}$ sucrose) and incubated for $30 \mathrm{~min}$ on ice to 895 release the periplasmic proteins. Soluble periplasmic 896 
proteins were isolated by two subsequent centrifugation steps. First, the supernatant was centrifuged for $15 \mathrm{~min}$ at $21,000 \mathrm{~g}$ to remove cellular debris. Subsequently, the supernatant was recentrifuged for $60 \mathrm{~min}$ at $120,000 \mathrm{~g}$ to remove denatured proteins and fragments of the cell membrane. The soluble periplasmic protein fraction was then loaded onto a 10-ml Strep-Tactin Sepharose column (IBA) pre-equilibrated with 5 bed volumes of buffer $\mathrm{W}$ (100 mM Tris- $\mathrm{HCl}$ and $100 \mathrm{mM} \mathrm{NaCl}, \mathrm{pH}$ 7.5). After the column was washed with 5 bed volumes of buffer $\mathrm{W}$, proteins bound to the affinity matrix were eluted with 3 bed volumes of buffer E (100 mM Tris-HCl, pH 7.5, $100 \mathrm{mM} \mathrm{NaCl}$ and $2.5 \mathrm{mM}$ desthiobiotin) from the column. UehA-containing fractions were collected in 3$\mathrm{ml}$ aliquots, and their protein content was analyzed by SDS-PAGE. UehA protein used for crystallization experiments was concentrated to approximately $12 \mathrm{mg} \mathrm{ml}^{-1}$ by using Vivaspin 4 (Vivascience, Hannover, Germany) concentrator columns (exclusion size $=10 \mathrm{kDa}$ ) in a buffer containing $10 \mathrm{mM}$ Tris- $\mathrm{HCl}, \mathrm{pH} 7.5$, and $10 \mathrm{mM} \mathrm{NaCl}$.

\section{Size-exclusion chromatography of UehA}

The oligomeric composition of the purified UehA protein was determined by size-exclusion chromatography on a Superdex 75 10/300 column (GE Healthcare, München, Germany). The column was equilibrated and run with $10 \mathrm{mM}$ Tris- $\mathrm{HCl}, \mathrm{pH} 7.5$, and $100 \mathrm{mM} \mathrm{NaCl}$, and the elution profile of proteins was monitored at a wavelength of $280 \mathrm{~nm}$ using an ÄKTA Purifier System (GE Healthcare). The following marker proteins were used: cytochrome $C(12.4 \mathrm{kDa})$, carbonic anhydrase $(29 \mathrm{kDa})$ and serum albumin (66 kDa) (Sigma-Aldrich).

\section{Determination of the dissociation constants of UehA for ectoine and 5-hydroxyectoine}

The UehA protein contains 14 tyrosine and 2 tryptophane residues, which allowed us to measure the binding of ectoine and 5-hydroxyectoine by the purified UehA protein through the use of an intrinsic tryptophantyrosine fluorescence-based binding assay. ${ }^{24,31,32}$ Fluorescence spectroscopy measurements were performed using a Cary Eclipse fluorescence photometer (VARIAN, Palo Alto, CA). The excitation wavelength was set to $280 \mathrm{~nm}$ (slit width $=5 \mathrm{~nm}$ ), and temperature was maintained at $25 \pm 1{ }^{\circ} \mathrm{C}$ using a circulating water bath. A UehA protein solution $(5 \mu \mathrm{g} / \mathrm{ml}$ in $10 \mathrm{mM}$ Tris- $\mathrm{HCl}, \mathrm{pH} 7.5$, and $10 \mathrm{mM}$ $\mathrm{NaCl}$ ) was mixed with either ectoine or 5-hydroxyectoine solution (in $10 \mathrm{mM}$ Tris- $\mathrm{HCl}, \mathrm{pH} 7.5$, and $10 \mathrm{mM} \mathrm{NaCl}$ ). The mixture was then incubated for $2 \mathrm{~min}$ to allow equilibration before the actual measurement. Emission spectra were monitored from 280 to $410 \mathrm{~nm}$ using the program Cary Eclipse Scan (VARIAN). Upon substrate binding, a decrease in the fluorescence intensity of UehA was detected, and this change in the emission spectrum was used to determine the $K_{\mathrm{d}}$ values for the UehA protein for either ectoine or 5-hydroxyectoine by plotting the peak area from 295 to $400 \mathrm{~nm}$ against the substrate concentration. For convenience, data were converted into fluorescence increase and treated as and analyzed by assuming a standard one-site binding model according to:

$$
F_{\text {cor }}=F_{0}+\left(\Delta F\left[\mathrm{~S}_{0}\right] /\left[\mathrm{S}_{0}\right]+K_{\mathrm{d}}\right)
$$

where $F_{\text {cor }}$ is the fluorescence intensity for a given substrate concentration, $F_{0}$ is the fluorescence intensity without substrate, $\Delta F$ is the maximal change in fluorescence intensity, $\left[\mathrm{S}_{0}\right]$ is the substrate concentration and $K_{\mathrm{d}}$ is the 959 binding constant. For all concentrations, the total substrate 960 concentration (bound and free substrates) was used in the 961 calculations. All $K_{\mathrm{d}}$ measurements of UehA for its 962 substrates ectoine and 5-hydroxyectoine represent the 963 average of at least three independent measurements, 964 with standard deviations.

\section{Crystallization}

Crystallization trials were carried out using the hang- 967 ing-drop vapor-diffusion method at $18{ }^{\circ} \mathrm{C}$. Homogenous 968 UehA protein was concentrated to $12 \mathrm{mg} \mathrm{ml}^{-1}$ prior to 969 crystallization. For crystallization of UehA with substrate, 970 $2 \mathrm{mM}$ ectoine was added to the protein solution $30 \mathrm{~min} 971$ prior to crystallization. Crystals were grown by mixing 972 protein solution with a reservoir solution containing 973 100 mM Na-citrate, pH 5.6-6.0, and 2.4-2.6 M ammonium 974 sulfate in a 1:1 ratio. Crystals normally grew in 5 to 10975 days. Suitable crystals were cryoprotected using mother 976 liquid supplemented with $30 \%(\mathrm{v} / \mathrm{v})$ ethylene glycol and 977 flash frozen in liquid nitrogen.

978

\section{Data collection and structure determination}

The ectoine dataset was collected at the ID23-2 beamline 980 at the ESRF. Detailed information on data collection 981 statistics are shown in Table 1. The optimal data collection 982 strategy was calculated using the program BEST. ${ }^{53}$ The 983 collected dataset was processed with the DENZO ${ }^{54}$ and 984 XDS program packages. The TeaA model (kindly pro- 985 vided by Dr. C. Ziegler, Max-Planck-Institute of Biophy- 986 sics, Frankfurt, Germany) was used as the template to 987 phase the UehA dataset at $2.9 \AA$ using the molecular 988 replacement program PHASER ${ }^{34}$ and further refined 989 using REFMAC5 ${ }^{55}$ and COOT. ${ }^{56}$ Dataset and refinement 990 statistics are summarized in Table 1.

991

\section{PDB accession code}

Coordinates for the UehA-ectoine complex have been 993 deposited in the PDB under accession code 3FXB.

Figure preparation

Figures of protein structures were prepared using 996 PyMOL\$.

\section{Databank searches, sequence and structural alignments}

Proteins that are homologous to the UehA, UehB, UehC 1000 and UspA proteins from S. pomeroyi DSS-3 were searched 1001 via the Web server of the DOE Joint Genome Institute ${ }^{2} 1002$ using the BLAST algorithm. ${ }^{46}$ The genome context of 1003 finished and unfinished microbial genomes in the vicinity 1004 of the $u e h A$ gene was assessed using the gene neighbor- 1005 hood toolll provided by the JGI Web server. Sequence 1006 alignments of proteins were performed using ClustalW ${ }^{47} 1007$ as implemented in the Vector NTI 10.0 software package 1008 (Invitrogen, Karlsruhe, Germany). Predictions of signal 1009

$\S$ http:/ / pymol.sourceforge.net/

|l http:/ /img.jgi.doe.gov/cgi-bin/pub/main.cgi 
1010 sequences of secreted proteins were conducted using the 1011 SignalP 3.0 Web server ${ }^{57}$ The prediction of the topology 1012 of integral membrane proteins was performed with 1013 SCAMPI, which is part of the consensus topology 1014 prediction Web server TOPCONS ${ }^{\text {a }}{ }^{30}$ Structure align1015 ments were performed using LSQMAN employing 1016 standard settings.

\section{Acknowledgements}

1018 This project was funded by grants from the 1019 University of Duesseldorf (L.S.), the University of 1020 Marburg, the Fonds der Chemischen Industrie and 1021 the Max-Planck-Institute for Terrestrial Microbiology 1022 (Marburg) (to E.B.). We are grateful to Dr. Christine 1023 Ziegler (Max-Planck-Institute of Biophysics, Frank1024 furt, Germany) for kindly providing us with the 1025 coordinates of the TehA crystal structure prior to 1026 publication. We are indebted to Margarete Schubert1027 Bätz for her excellent support in the early stages of 1028 the project and to Dr. Sacha Popov for his support 1029 during data collection at the ESRF beamline ID-23 1030 (Grenoble, France) as well as Dr. Paul Tucker and Dr. 1031 Mathew Grooves at the BW7A beamline (EMBL 1032 Outstation Hamburg, Hamburg, Germany). We 1033 acknowledge support of in-house facilities by the 1034 NRW Research School BioStruct, and we also thank 1035 Dr. Thomas Schwarz from bitop AG (Witten, 1036 Germany) for the generous gift of ectoine and 51037 hydroxyectoine and Dr. Mohamed Jebbar (Univer1038 sity of Rennes, Rennes, France) for kindly providing 1039 us with radiolabeled ectoine.

\section{Supplementary Data}

1041 Supplementary data associated with this article 1042 can be found, in the online version, at doi:10.1016/j. 1043 jmb.2009.03.077 type of periplasmic permease. Biochem. Soc. Trans. 21, 1060 343S.

5. Forward, J. A., Behrendt, M. C., Wyborn, N. R., Cross, 1062 R. \& Kelly, D. J. (1997). TRAP transporters: a new 1063 family of periplasmic solute transport systems 1064 encoded by the dctPQM genes of Rhodobacter capsula- 1065 tus and by homologs in diverse Gram-negative 1066 bacteria. J. Bacteriol. 179, 5482-5493. 1067

6. Kelly, D. J. \& Thomas, G. H. (2001). The tripartite ATP- 1068 independent periplasmic (TRAP) transporters of bac- 1069 teria and archaea. FEMS Microbiol. Rev. 25, 405-424. 1070

7. Rabus, R., Jack, D. L., Kelly, D. J. \& Saier, M. H., Jr 1071 (1999). TRAP transporters: an ancient family of extra- 1072 cytoplasmic solute-receptor-dependent secondary 1073 active transporters. Microbiology, 145(Pt 12), 3431-3445. 1074

8. Mulligan, C., Kelly, D. J. \& Thomas, G. H. (2007). 1075 Tripartite ATP-independent periplasmic transporters: 1076 application of a relational database for genome-wide 1077 analysis of transporter gene frequency and organiza- 1078 tion. I. Mol. Microbiol. Biotechnol. 12, 218-226. 1079

9. Mulligan, C., Geertsma, E. R., Severi, E., Kelly, D. J., 1080 Poolman, B. \& Thomas, G. H. (2009). The substrate- 1081 binding protein imposes directionality on an electro- 1082 chemical sodium gradient-driven TRAP transporter. 1083 Proc. Natl Acad. Sci. USA. 1084

10. Shaw, J. G., Hamblin, M. J. \& Kelly, D. J. (1991). 1085 Purification, characterization and nucleotide sequence 1086 of the periplasmic C4-dicarboxylate-binding protein 1087 (DctP) from Rhodobacter capsulatus. Mol. Microbiol. 5, 1088 3055-3062.

1089

11. Walmsley, A. R., Shaw, J. G. \& Kelly, D. J. (1992). The 1090 mechanism of ligand binding to the periplasmic C4- 1091 dicarboxylate binding protein (DctP) from Rhodobacter 1092 capsulatus. J. Biol. Chem. 267, 8064-8072. 1093

12. Johnston, J. W., Coussens, N. P., Allen, S., Houtman, 1094 J. C., Turner, K. H., Zaleski, A. et al. (2008). 1095 Characterization of the $N$-acetyl-5-neuraminic acid- 1096 binding site of the extracytoplasmic solute receptor 1097 (SiaP) of nontypeable Haemophilus influenzae strain 1098 2019. J. Biol. Chem. 283, 855-865. 1099

13. Gonin, S., Arnoux, P., Pierru, B., Lavergne, J., Alonso, 1100 B., Sabaty, M. \& Pignol, D. (2007). Crystal structures of 1101 an extracytoplasmic solute receptor from a TRAP 1102 transporter in its open and closed forms reveal a helix- 1103 swapped dimer requiring a cation for alpha-keto acid 1104 binding. BMC Struct. Biol. 7, 11.

14. Rucktooa, P., Antoine, R., Herrou, J., Huvent, I., Locht, 1106 C., Jacob-Dubuisson, F. et al. (2007). Crystal structures 1107 of two Bordetella pertussis periplasmic receptors con- 1108 tribute to defining a novel pyroglutamic acid binding 1109 DctP subfamily. J. Mol. Biol. 370, 93-106. 1110

15. Kuhlmann, S. I., Terwisscha van Scheltinga, A. C., 1111 Bienert, R., Kunte, H. J. \& Ziegler, C. (2008). $1.55 \AA 1112$ structure of the ectoine binding protein TeaA of the 1113 osmoregulated TRAP-transporter TeaABC from Halo- 1114 monas elongata. Biochemistry, 47, 9475-9485. 1115

16. Cuneo, M. J., Changela, A., Miklos, A. E., Beese, L. S., 1116 Krueger, J. K. \& Hellinga, H. W. (2008). Structural 1117 analysis of a periplasmic binding protein in the 1118 tripartite ATP-independent transporter family reveals 1119 a tetrameric assembly that may have a role in ligand 1120 transport. J. Biol. Chem. 283, 32812-32820.

17. Muller, A., Severi, E., Mulligan, C., Watts, A. G., Kelly, 1122 D. J., Wilson, K. S. et al. (2006). Conservation of 1123 structure and mechanism in primary and secondary 1124 transporters exemplified by SiaP, a sialic acid binding 1125 virulence factor from Haemophilus influenzae. I. Biol. 1126 Chem. 281, 22212-22222.

18. Wilkinson, J. \& Verschueren, K. H. G. (2003). Crystal 1128 
structures of periplasmic solute-binding proteins in $\mathrm{ABC}$ transport complexes illuminate their function. In ABC Proteins: From Bacteria to Man (Holland, I. B., Cole, S. P. C., Kuchler, K. \& Higgins, C. F., eds), pp. 187-208, Academic Press (Elsevier Science), London, UK.

19. Mao, B., Pear, M. R., McCammon, J. A. \& Quiocho, F. A. (1982). Hinge-bending in L-arabinose-binding protein. The "Venus's-flytrap" model. J. Biol. Chem. 257, 1131-1133.

20. Moran, M. A., Buchan, A., Gonzalez, J. M., Heidelberg, J. F., Whitman, W. B., Kiene, R. P. et al. (2004). Genome sequence of Silicibacter pomeroyi reveals adaptations to the marine environment. Nature, 432, 910-913.

21. Brinkhoff, T., Giebel, H. A. \& Simon, M. (2008). Diversity, ecology, and genomics of the Roseobacter clade: a short overview. Arch. Microbiol. 189, 531-539.

22. Kempf, B. \& Bremer, E. (1998). Uptake and synthesis of compatible solutes as microbial stress responses to high-osmolality environments. Arch. Microbiol. 170, 319-330.

23. Grammann, K., Volke, A. \& Kunte, H. J. (2002). New type of osmoregulated solute transporter identified in halophilic members of the bacteria domain: TRAP transporter TeaABC mediates uptake of ectoine and hydroxyectoine in Halomonas elongata DSM $2581^{(\mathrm{T})}$. J. Bacteriol. 184, 3078-3085.

24. Hanekop, N., Hoing, M., Sohn-Bosser, L., Jebbar, M., Schmitt, L. \& Bremer, E. (2007). Crystal structure of the ligand-binding protein EhuB from Sinorhizobium meliloti reveals substrate recognition of the compatible solutes ectoine and hydroxyectoine. J. Mol. Biol. 374, 1237-1250.

25. Kuhlmann, A. U., Bursy, J., Gimpel, S., Hoffmann, T. \& Bremer, E. (2008). Synthesis of the compatible solute ectoine in Virgibacillus pantothenticus is triggered by high salinity and low growth temperature. Appl. Environ. Microbiol. 74, 4560-4563.

26. Kuhlmann, A. U. \& Bremer, E. (2002). Osmotically regulated synthesis of the compatible solute ectoine in Bacillus pasteurii and related Bacillus spp. Appl. Environ. Microbiol. 68, 772-783.

27. Bursy, J., Pierik, A. J., Pica, N. \& Bremer, E. (2007). Osmotically induced synthesis of the compatible solute hydroxyectoine is mediated by an evolutionarily conserved ectoine hydroxylase. J. Biol. Chem. 282, 31147-31155.

28. Bursy, J., Kuhlmann, A. U., Pittelkow, M., Hartmann, H., Jebbar, M., Pierik, A. J. \& Bremer, E. (2008) Synthesis and uptake of the compatible solutes ectoine and 5-hydroxyectoine by Streptomyces coelicolor A3(2) in response to salt and heat stress. Appl. Environ. Microbiol. 74, 7286-7296.

29. Jebbar, M., Sohn-Bosser, L., Bremer, E., Bernard, T. \& Blanco, C. (2005). Ectoine-induced proteins in Sinorhizobium melilot $i$ include an ectoine ABC-type transporter involved in osmoprotection and ectoine catabolism. J. Bacteriol. 187, 1293-1304.

30. Bernsel, A., Viklund, H., Falk, J., Lindahl, E., von Heijne, G. \& Elofsson, A. (2008). Prediction of membrane-protein topology from first principles. Proc. Natl Acad. Sci. USA, 105, 7177-7181.

31. Horn, C., Sohn-Bosser, L., Breed, J., Welte, W., Schmitt, L. \& Bremer, E. (2006). Molecular determinants for substrate specificity of the ligand-binding protein OpuAC from Bacillus subtilis for the compatible solutes glycine betaine and proline betaine. J. Mol. Biol. 357, 592-606.

32. Oswald, C., Smits, S. H., Hoing, M., Sohn-Bosser, L.
Dupont, L., Le Rudulier, D. et al. (2008). Crystal 1198 structures of the choline/acetylcholine substrate 1199 binding protein ChoX from Sinorhizobium meliloti in 1200 the liganded and unliganded closed states. J. Biol. 1201 Chem. 283, 32848-32859.

1202

33. Kabsch, W. (1993). Automatic processing of rotation 1203 diffraction data from crystals of initially unknown 1204 symmetry and cell constants. J. Appl. Crystallogr. 26, 1205 795-800.

1206

34. McCoy, A. J., Grosse-Kunstleve, R. W., Adams, P. D., 1207 Winn, M. D., Storoni, L. C. \& Read, R. J. (2007). Phaser 1208 crystallographic software. J. Appl. Crystallogr. 40, 1209 658-674.

1210

35. Fukami-Kobayashi, K., Tateno, Y. \& Nishikawa, K. 1211 (1999). Domain dislocation: a change of core structure 1212 in periplasmic binding proteins in their evolutionary 1213 history. J. Mol. Biol. 286, 279-290.

1214

36. Street, T. O., Bolen, D. W. \& Rose, G. D. (2006). A 1215 molecular mechanism for osmolyte-induced protein 1216 stability. Proc. Natl Acad. Sci. USA, 103, 13997-14002. 1217

37. Smits, S. H., Hoing, M., Lecher, J., Jebbar, M., 1218 Schmitt, L. \& Bremer, E. (2008). The compatible 1219 solute-binding protein OpuAC from Bacillus subtilis: 1220 ligand-binding, site directed mutagenesis and crys- 1221 tallographic studies. J. Bacteriol. 1222

38. Schiefner, A., Holtmann, G., Diederichs, K., Welte, W. 1223 \& Bremer, E. (2004). Structural basis for the binding of 1224 compatible solutes by ProX from the hyperthermo- 1225 philic archaeon Archaeoglobus fulgidus. J. Biol. Chem. 1226 279, 48270-48281.

39. Schiefner, A., Breed, J., Bosser, L., Kneip, S., Gade, J., 1228 Holtmann, G. et al. (2004). Cation-pi interactions as 1229 determinants for binding of the compatible solutes 1230 glycine betaine and proline betaine by the periplasmic 1231 ligand-binding protein ProX from Escherichia coli. 1232 J. Biol. Chem. 279, 5588-5596. 1233

40. Mahmood, N. A., Biemans-Oldehinkel, E., Patzlaff, J. S., 1234 Schuurman-Wolters, G. K. \& Poolman, B. (2006). Ion 1235 specificity and ionic strength dependence of the 1236 osmoregulatory ABC transporter OpuA. J. Biol. Chem. 1237 281, 29830-29839.

41. Ott, V., Koch, J., Spate, K., Morbach, S. \& Kramer, R. 1239 (2008). Regulatory properties and interaction of the C- 1240 and N-terminal domains of BetP, an osmoregulated 1241 betaine transporter from Corynebacterium glutamicum. 1242 Biochemistry, 47, 12208-12218.

42. Culham, D. E., Vernikovska, Y., Tschowri, N., Keates, 1244 R. A., Wood, J. M. \& Boggs, J. M. (2008). Periplasmic 1245 loops of osmosensory transporter ProP in Escherichia 1246 coli are sensitive to osmolality. Biochemistry, 47, 1247 13584-13593.

43. Oldham, M. L., Khare, D., Quiocho, F. A., Davidson, 1249 A. L. \& Chen, J. (2007). Crystal structure of a catalytic 1250 intermediate of the maltose transporter. Nature, 450, 1251 515-521.

44. Hollenstein, K., Frei, D. C. \& Locher, K. P. (2007). 1253 Structure of an ABC transporter in complex with its 1254 binding protein. Nature, 446, 213-216. 1255

45. Hvorup, R. N., Goetz, B. A., Niederer, M., Holenstein, 1256 K., Perozo, E. \& Locher, K. P. (2007). Asymmetry in the 1257 structure of the ABC transporter-binding protein 1258 complex BtuCD-BtuF. Science, 317, 1387-1390. 1259

46. Altschul, S. F., Madden, T. L., Schaffer, A. A., Zhang, J., 1260 Zhang, Z., Miller, W. \& Lipman, D. J. (1997). Gapped 1261 BLAST and PSI-BLAST: a new generation of protein 1262 database search programs. Nucleic Acids Res. 25, 1263 3389-3402.

47. Thompson, J. D., Higgins, D. G. \& Gibson, T. J. (1994). 1265 CLUSTAL W: improving the sensitivity of progressive 1266 
multiple sequence alignment through sequence weighting, position-specific gap penalties and weight matrix choice. Nucleic Acids Res. 22, 4673-4680.

48. Kvint, K., Nachin, L., Diez, A. \& Nystrom, T. (2003). The bacterial universal stress protein: function and regulation. Curr. Opin. Microbiol. 6, 140-145.

49. Jebbar, M., Talibart, R., Gloux, K., Bernard, T. \& Blanco, C. (1992). Osmoprotection of Escherichia coli by ectoine: uptake and accumulation characteristics. J. Bacteriol. 174, 5027-5035.

50. Gonzalez, J. M., Covert, J. S., Whitman, W. B., Henriksen, J. R., Mayer, F., Scharf, B. et al. (2003). Silicibacter pomeroyi sp. nov. and Roseovarius nubinhibens sp. nov., dimethylsulfoniopropionate-demethylating bacteria from marine environments. Int. J. Syst. Evol. Microbiol. 53, 1261-1269.

51. Baumann, P., Baumann, L. \& Mandel, M. (1971). Taxonomy of marine bacteria: the genus Beneckea. J. Bacteriol. 107, 268-294.

52. Miller, J. H. (1992). A Short Course in Bacterial Genetics.
A Laboratory Manual and Handbook for Escherichia coli 1287 and Related Bacteria, Cold Spring Harbor Laboratory, 1288 Cold Spring Harbor, NY.

53. Popov, A. N. \& Bourenkov, G. P. (2003). Choice of data- 1290 collection parameters based on statistic modelling. Acta 1291 Crystallogr., Sect. D: Biol. Crystallogr. 59, 1145-1153. 1292

54. Otwinowski, Z. \& Minor, W. (1997). Processing of 1293 X-ray diffraction data collected in oscillation mode. 1294 In Methods Enzymology (Carter, C. W. \& Sweet, R. M., 1295 eds), Vol. 276, Academic Press, London, UK. 1296

55. Murshudov, G., Vagin, A. A. \& Dodson, E. J. (1997). 1297 Refinement of macromolecular structures by the 1298 maximum-likelihood method. Acta Crystallogr., Sect. 1299 D: Biol. Crystallogr. 53, 240-255.

56. Emsley, P. \& Cowtan, K. (2004). Coot: model-building 1301 tools for molecular graphics. Acta Crystallogr., Sect. D: 1302 Biol. Crystallogr. 60, 2126-2132.

57. Bendtsen, J. D., Nielsen, H., von Heijne, G. \& Brunak, 1304 S. (2004). Improved prediction of signal peptides: 1305 SignalP 3.0. J. Mol. Biol. 340, 783-795. 